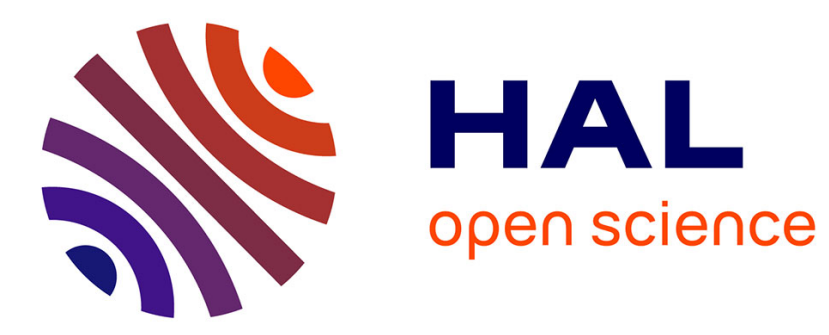

\title{
Comparison of piezoelectronic networks acting as distributed vibration absorbers
}

\author{
Corrado Maurini, Francesco Dell'Isola, Dionisio del Vescovo
}

\section{To cite this version:}

Corrado Maurini, Francesco Dell'Isola, Dionisio del Vescovo. Comparison of piezoelectronic networks acting as distributed vibration absorbers. Mechanical Systems and Signal Processing, 2004, pp.29. hal-00502098

\section{HAL Id: hal-00502098 \\ https://hal.science/hal-00502098}

Submitted on 13 Jul 2010

HAL is a multi-disciplinary open access archive for the deposit and dissemination of scientific research documents, whether they are published or not. The documents may come from teaching and research institutions in France or abroad, or from public or private research centers.
L'archive ouverte pluridisciplinaire HAL, est destinée au dépôt et à la diffusion de documents scientifiques de niveau recherche, publiés ou non, émanant des établissements d'enseignement et de recherche français ou étrangers, des laboratoires publics ou privés. 


\title{
Comparison of piezoelectronic networks acting as distributed vibration absorbers
}

\author{
Corrado Maurini ${ }^{\mathrm{a}, \mathrm{c}}$, Francesco dell'Isola ${ }^{\mathrm{b}, *}$, Dionisio Del Vescovo ${ }^{\mathrm{a}}$ \\ a Dipartimento di Meccanica e Aeronautica, Università di Roma "La Sapienza", Via Eudossiana 18, Roma I-00184, Italy \\ ${ }^{\mathrm{b}}$ Dipartimento di Ingegneria Strutturale e Geotecnica, Università di Roma "La Sapienza”, Via Eudossiana 18, \\ Roma I-00184, Italy \\ ${ }^{\mathrm{c}}$ ESM Department, Virginia Polytechnic Institute and State University, Blacksburg VA 24061, USA
}

\begin{abstract}
Electric vibration absorbers made of distributed piezoelectric devices for the control of beam vibrations are studied. The absorbers are obtained by interconnecting an array of piezoelectric transducers uniformly distributed on a beam with different modular electric networks. Five different topologies are considered and their damping performance is analysed and compared. Their optimal parameters are found by adopting a criterion for critical damping of $\bar{k}$-waves: the parameters are suitably chosen to have the quickest temporal vibration decay for a single wave number $\bar{k}$. The analysis is based on homogenized models of the modular piezo-electromechanical systems, i.e. they are regarded as continuous systems by assuming that the number of modules per unit length is high enough with respect to the considered wave numbers. Calling $\bar{k}$-absorbers the corresponding optimal absorbers, we show that: (i) $\bar{k}$-waves are damped in $\bar{k}$-absorbers with an optimal decay time which is independent of the absorber interconnecting topology, while it depends only on the piezoelectric coupling coefficient; (ii) the efficiency of $\bar{k}$-absorbers depends significantly on the absorber interconnecting topology for $k$ different from $\bar{k}$; (iii) one of the proposed absorbers (which is made of a fourth-order electric transmission line with a second-order electric dissipation) equally performs for all the wave numbers and accomplishes an effective multi-modal damping for the mechanically forced response; (iv) the optimal values of the electric parameters differently depend on the number $n$ of used circuit modules for different interconnecting topologies and, in particular, the optimal inductance per module needed in a fourth-order electric transmission line is proportional $1 / n^{3}$. (C) 2003 Elsevier Ltd. All rights reserved.
\end{abstract}

*Corresponding author.

E-mail address: francesco.dellisola@uniroma1.it (F. dell'Isola).

0888-3270/\$ - see front matter (C) 2003 Elsevier Ltd. All rights reserved. doi:10.1016/S0888-3270(03)00082-7 


\section{Introduction}

Passive vibration control can be achieved by coupling a mechanical structure $S$ to an auxiliary dissipative system $S^{\prime}$ by means of an appropriate transduction device $T$. Once the properties of $T$ are given, a proper design of the auxiliary system $S^{\prime}$ allows enhancement of the energy exchange between $S$ and $S^{\prime}$ and the energy dissipation in $S^{\prime}$ by exploiting an internal resonance phenomenon in $\left(S, T, S^{\prime}\right)$. In the technical literature the additional system $S^{\prime}$ is called dynamic vibration absorber. For example, the flexural vibrations of an Euler beam can be controlled by connecting it to a proper spring-mass-damper system (see [1]). The same effect can be achieved by bonding on the beam a piezoelectric transducer shunted to a resistor and an inductor, obtaining the so-called resonant shunted piezoelectric transducer (see [2-4]). Indeed, the resistor and the inductor, together with the capacitance of the piezoelectric transducer, form an RLC circuit piezoelectrically coupled to a vibration mode of the beam. An efficient electrical dissipation of the mechanical vibration energy can be achieved thanks to the present technology of piezoelectric materials, which offer the possibility of producing transducers able to efficiently convert mechanical into electrical energy (and vice versa).

The vibration control of a continuum system $S$, like a beam or a plate, obtained by ane-degreeof-freedom vibration absorber $S^{\prime}$ and a localized single transduction device $T$, implies several drawbacks. Indeed, in this case $S^{\prime}$ can be tuned to one vibration mode of $S$ only, and the energy exchange between $S$ and $S^{\prime}$ is effective in a narrow frequency band (unimodal damping). Moreover, the problem of the optimal positioning of the transduction device for each particular geometrical and dynamic condition arises. In addition, since the energy flow between $S$ and $S^{\prime}$ is concentrated in a small space region, the single transducer can be overstressed. Some variations to the single resonant shunted piezoelectric transducer were studied to overcome some of these drawbacks. In [3] the optimal spatial placement of several resonant shunted piezoelectric transducers, each one tuned to damp a different vibration mode, is studied. Other authors (see e.g. [5]) propose to shunt a single piezoelectric transducer with a multi-degree-of-freedom electric network, resonating at more than one frequency, in order to efficiently couple the absorber to multiple mechanical modes.

An alternative approach suggests to couple the continuum system $S$ to a distributed auxiliary system $S^{\prime}$ with distributed transduction devices. In this case we call $S^{\prime}$ distributed vibration absorber. In [6-8], it is proposed to use distributed piezoelectric transducers to couple mechanical structures to distributed electric networks. In this way it is possible to synthesize an electromechanical medium, the damping properties of which are controlled by variation of electric parameters. In particular, it is shown that, when mechanical structures like bars, beams or plates are coupled with electric systems possessing analogous dynamic properties (e.g. dispersion relations), an efficient wideband electromechanical energy exchange is obtained. For example, if an array of piezoelectric transducers bonded on a bar is interconnected by floating inductors, all the vibration modes of the bar can be optimally coupled with those of a second-order electric transmission line. In [9] the problem of synthesizing electric networks providing a multi-modal energy exchange with beams and plates is addressed and some solutions are proposed. More recently, the idea of distributed passive control was investigated in [10], where wave propagation in a periodic electromechanical medium consisting of a beam with an array of resonant independently shunted piezoelectric transducers is studied. In [11] it is proposed to embed 
piezoelectric fibres in a resistive epoxy matrix to obtain a highly dissipative electromechanical material. The mathematical problem of continuum modelling periodic media including piezoelectric transducers and electronic circuits is tackled in [12].

The construction of the piezoelectric devices described above presents a technical problem: due to the capacitance values of the piezoelectric transducers, electric networks resonating in the band of the lower structural eigenfrequencies require high-value inductors (up to thousands of Henry). For this reason, a completely passive realization of these devices is not conceivable and electronic active filters simulating inductive impedances must be employed, thus implicating important consequences. On one hand, the use of electronic circuits allows the realization of the required electric networks by light and small integrated devices which can be embedded into the structure; moreover, the properties of those electronic circuits can be easily controlled by external commands and adaptive piezoelectromechanical structures can be conceived (see e.g. [13,14]). On the other hand, the electronic circuits require an external power supply and, although only a low power is needed, the advantages of a completely passive control device are partially lost.

In this paper, we exploit the idea of distributed piezoelectric transduction for the vibration control of beams by means of dissipative electric networks. We analyse and compare the optimal damping performance achievable by interconnecting an array of piezoelectric transducers through different circuit topologies. The circuit topologies are synthesized in order to obtain, together with the piezoelectric transducers, electric networks emulating the behaviour of dynamic vibration absorbers with assigned equations of motion. The whole analysis is based on one-dimensional continuum models of the modular electromechanical systems.

The exposure is organized as follows. In Section 2, the electromechanical systems are briefly presented. In Section 3, the corresponding mathematical models are derived. We start by presenting a micro-model of the basic module of a modular system consisting of a beam and an array of piezoelectric transducers with non-local electric interactions. Hence, a macrohomogenized model of that system is introduced. The corresponding constitutive properties are deduced by assuming a mapping between the micro- and macro-kinematics and the equivalence of the expended powers. The set of partial differential equations describing the systems, composed of the beam and one of the five electric vibration absorbers, is obtained by particular choices of the constitutive parameters. In Section 4, for each interconnecting topology, the dynamics of the resulting electromechanical structures is studied and the electric parameters for an optimal damping of $\bar{k}$-waves (i.e. waves with a fixed wave number $\bar{k}$ ) are found. Hence, we analyse the damping performance for wave numbers $k$ differing from the value $\bar{k}$ for which the absorbers were designed. Finally, we compare the behaviour of the five different circuit configurations (which are obtained by inserting resistors and inductors in different circuit branches). As a numerical example, we study the vibration damping of a rectangular cross-section aluminium beam on which pairs of piezoelectric transducers are bonded, aiming at a preliminary design of experimental prototypes of the proposed devices.

\section{Description of considered electromechanical systems}

A pair of piezoelectric transducers in bimorph configuration (Fig. 1) provides a coupling between the flexural vibrations of the beam and the dynamics of a proper electric circuit. As an extension, a 


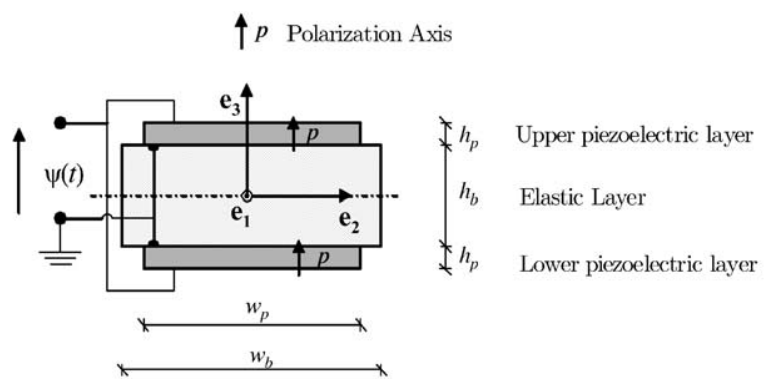

Fig. 1. Three-layered beam element with piezoelectric transducers in bimorph configuration: cross-section. The upper and lower piezoelectric transducers consist of layers of thickness-polarized piezoelectric ceramic with electroded upper and lower surfaces. The electrodes of the two layers are interconnected in parallel and in counter phase in order to couple the applied voltage to the beam bending (and vice versa).

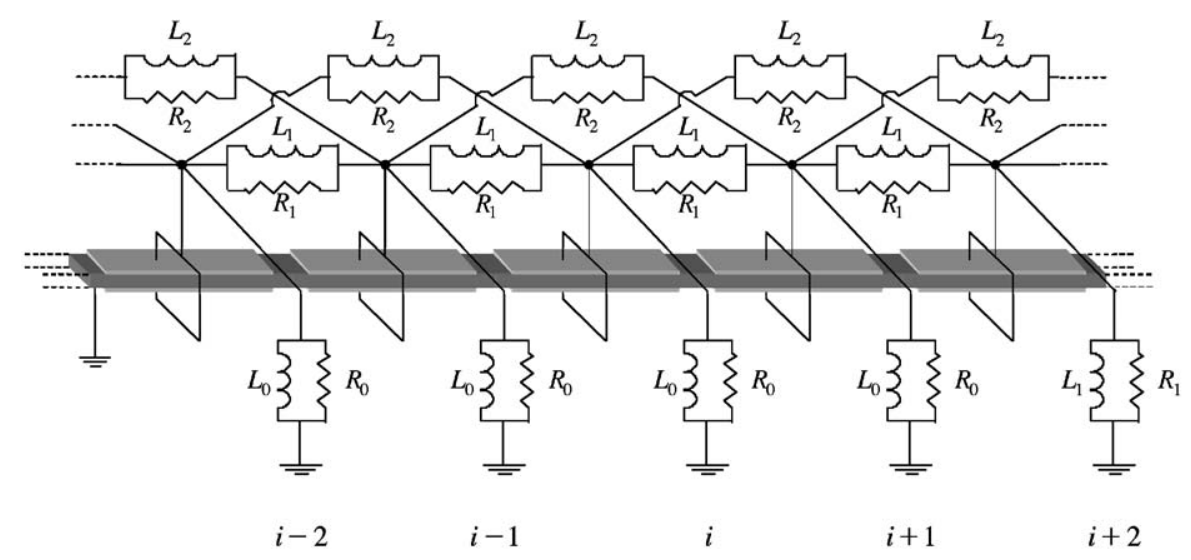

Fig. 2. Generic modular PEM beam.

modular piezo-electro mechanical (PEM) beam like the one in Fig. 2 provides an electromechanical medium in which the beam vibrations are coupled with a distributed electric network. The general module of the PEM beam is composed of an elastic beam element, piezoelectric transducers, inductances and resistances as shown in Fig. 3. Each cell is electrically interacting with the nearest and the next-to-nearest neighbours through a parallel RL impedance (the electric network corresponds to an electric lattice with next-to-nearest neighbour interactions, as defined in [16]). In such a system the equivalent capacitances of the piezoelectric transducers together with the interconnecting RL network form an electric waveguide which is piezoelectrically coupled to the beam oscillations. For dynamic phenomena characterized by sufficiently long wavelengths, the modular system can be modelled as a micro-structured electromechanical continuum, the constitutive properties of which are controlled by acting on electric parameters. With this approach the lumped electric network is modelled as a continuous system and the dynamics of the whole PEM beam is described by a set of two coupled differential equations. If an Euler model is accepted, the beam flexural vibrations are governed by a 


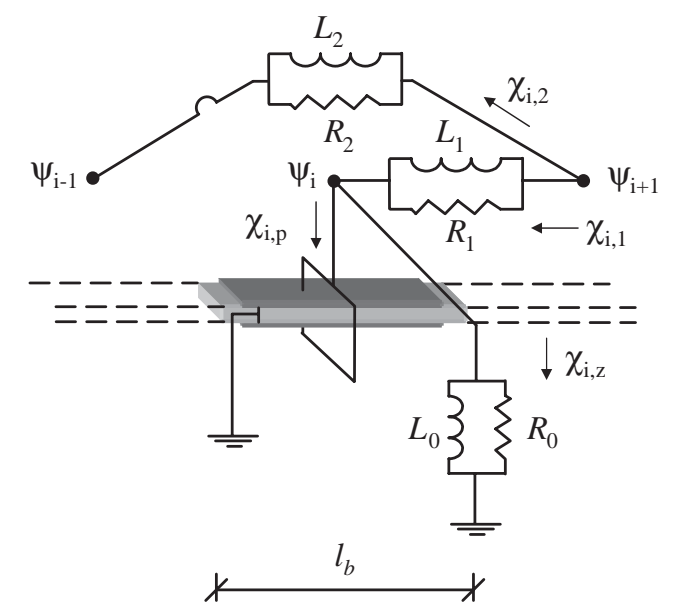

Fig. 3. Generic modular PEM beam: basic module.

fourth-order differential equation of the type (here and henceforth $\square^{\prime}=\partial / \partial x, \dot{\square}=\partial / \partial t$ )

$$
U^{\mathrm{IV}}(X, t)+\frac{1}{c_{b}^{2}} \ddot{U}(X, t)=0,
$$

where $U(X, t)$ is the transverse displacement of the beam axis at the point labelled by the abscissa $X$ and $c_{b}$ is the speed of the free bending waves. In the following, we show that suitable choices of the electric parameters of the circuit scheme in Fig. 3 lead to systems in which the beam vibrations (1) are piezoelectrically coupled to the electric networks characterized by the following homogenized evolution equations for the electric flux-linkage $\Psi$ (the electric flux-linkage is defined as the time primitive of the electric potential):

(Z, Z)-network: zeroth-order network with zeroth-order dissipation,

$$
\ddot{\Psi}(X, t)+\delta_{0} \dot{\Psi}(X, t)+\beta_{0} \Psi(X, t)=0 .
$$

(S, Z)-network: second-order network with zeroth-order dissipation,

$$
\ddot{\Psi}(X, t)+\delta_{0} \dot{\Psi}(X, t)-\beta_{2} \Psi^{\prime \prime}(X, t)=0 .
$$

$(\mathrm{S}, \mathrm{S})$-network: second-order network with second-order dissipation,

$$
\ddot{\Psi}(X, t)-\delta_{2} \dot{\Psi}^{\prime \prime}(X, t)-\beta_{2} \Psi^{\prime \prime}(X, t)=0 .
$$

$(\mathrm{F}, \mathrm{Z})$-network: fourth-order network with zeroth-order dissipation,

$$
\ddot{\Psi}(X, t)+\delta_{0} \dot{\Psi}(X, t)+\beta_{4} \Psi^{\mathrm{IV}}(X, t)=0 .
$$

(F, S)-network: fourth-order network with second-order dissipation,

$$
\ddot{\Psi}(X, t)-\delta_{2} \dot{\Psi}^{\prime \prime}(X, t)+\beta_{4} \Psi^{\mathrm{IV}}(X, t)=0 .
$$

The (.,.)-network nomenclature above refers to the order of spatial derivatives appearing in the third and second term of the left-hand side of the equations of motion (2). For example, the (F, S)network is characterized by a fourth-order spatial derivative on $\Psi$ and a second-order spatial 


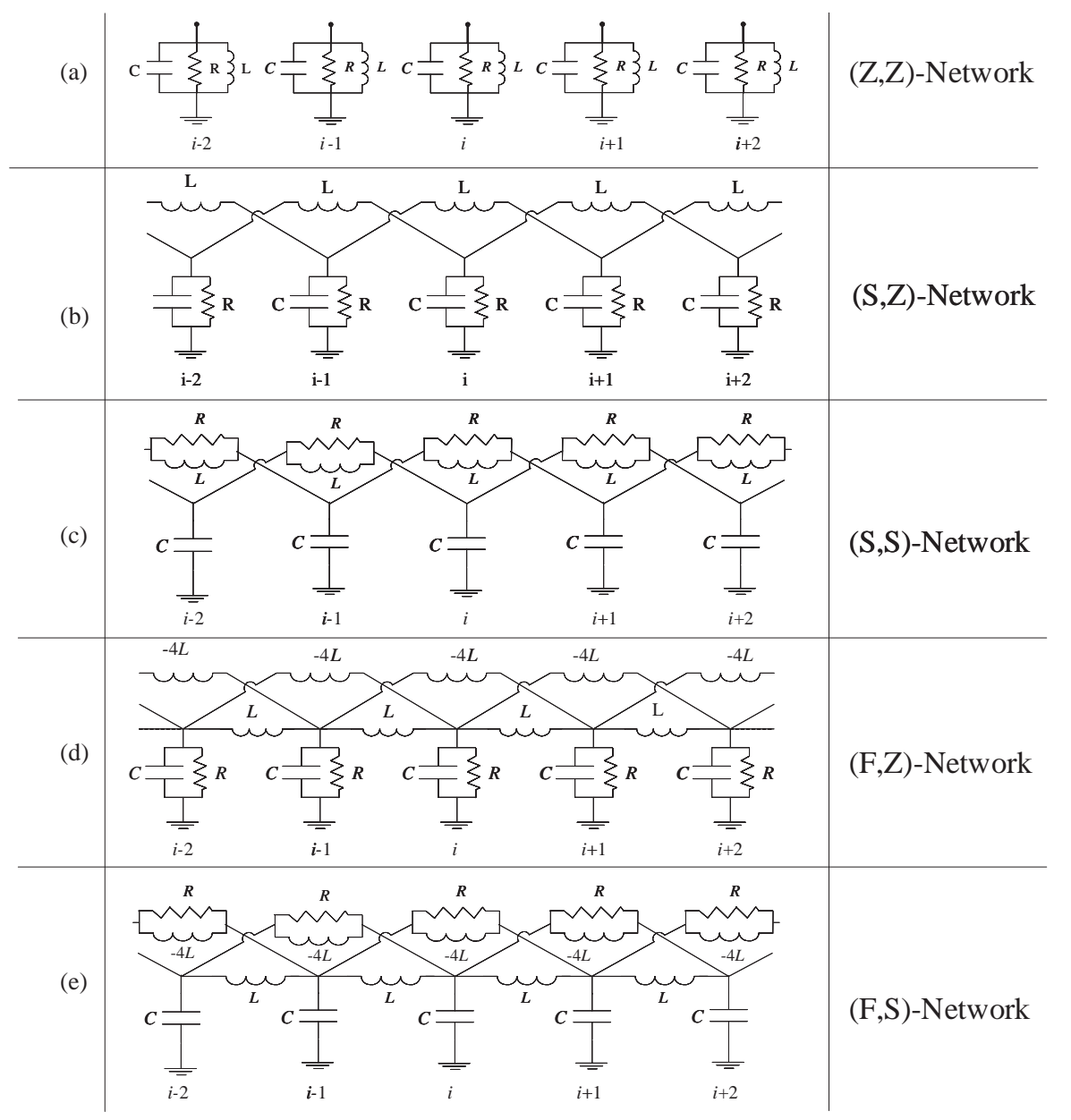

Fig. 4. Circuit schemes of the modular lumped electric networks corresponding to Eqs. (2a)-(2e).

derivative on $\dot{\Psi}(Z \equiv 0$ th, $\equiv 2$ nd $F \equiv 4$ th). The lumped electric network modelled by Eqs. (2) are reported explicitly in Fig. 4. The related PEM beams in which mechanical and electrical oscillations are piezoelectrically coupled are obtained by replacing the capacitances with piezoelectric transducers uniformly distributed along the beam. In the following, after that continuum models of the electromechanical systems are derived, we study the electromechanical coupling between the mechanical beam and the five electric networks (2). By regarding the latter as electric absorbers for the beam vibrations, optimal damping and tuning parameters are found and the corresponding damping performances are compared.

The correspondence between the lumped electric networks in Fig. 4 and the equations of motion (2) is obtained by assuming an approximation method based on a centred finite difference scheme. If different discretization methods are used, different lumped electric networks governed by the same equations (2) can be found. The circuital topologies in Fig. 4 require negative inductors for the fourth-order networks $(F, Z)$ and $(F, S)$. These negative inductors must be 
synthesized by means of active electronic circuits (see [9] for further details). An alternative solution for the synthesis of the fourth-order transmission lines is presented in [17], where the use of negative inductors is avoided (similar synthesis problems were studied to find electric analogs for mechanical systems in order to perform analog computing [15]).

\section{Mathematical modelling}

In this section homogenized equations of motion for the modular PEM beam in Fig. 2 are derived. We introduce two mathematical descriptions of such a system:

(a) A micro-model, which regards the system as an axially non-homogeneous layered piezoelectric beam coupled to a lumped electric network.

(b) A macro-model, which regards the system as an electromechanical micro-structured continuum the constitutive properties of which are determined by those of the basic module.

The micro-model describes the system dynamics by a partial differential equation coupled to a set of ordinary differential equations, while the macro-model furnishes a system of two coupled PDEs. In the following sections, only the homogenized equations of motion of the macro-model are considered for the dynamic analysis. The description at micro-level is presented because it is necessary to rationally deduce the macro-constitutive properties. To this aim, the micro-macroidentification method in virtual powers is adopted [18]. This method starts from the weak formulation of evolutionary problems based on D'Alembert principle of virtual powers (for its modern presentation see [19,20]). In this approach, a kinematical description of the system is assumed, and then the model is expressed by means of virtual power linear functionals. They associate - once a state of the system is fixed - each admissible virtual velocity field to the scalar quantities representing the corresponding powers expended by applied dynamic actions. A weak form of the balance equations of a given system is directly derived by imposing the equality between external and internal powers for each admissible virtual velocity field. Under suitable regularity conditions, the weak balance equations can be proven to be equivalent to a set of differential equations, which are their strong counterpart.

The identification method is based on the following procedure:

(1) The kinematics of micro- and macro-models is specified and admissible micro- and macrovelocity fields are introduced.

(2) A kinematical map is chosen and a precise micro-state is associated to each macrokinematical state.

(3) An expression for expended powers at the micro-level is postulated in terms of admissible test micro-velocity fields: the use of the kinematical map allows the definition of macrogeneralized forces and the derivation of macro-balance and macro-constitutive equations in terms of the micro-ones.

Obviously, the efficiency of the macro-model in approximating the micro-one relies on the choice of the kinematical map. In the present paper, instead of attempting to rigorously determine the conditions under which the kinematical map is reliable, we simply develop some heuristic arguments. In particular, we assume as kinematical map a local expansion of the 
micro-kinematics in terms of the macro-one, which is valid when the ratio between the basic module length $l_{b}$ and the characteristic length $\lambda$ of the considered phenomena is small. For further details about the adopted method, we refer to [18,21].

\subsection{Kinematics}

Let us consider a PEM beam composed of $n_{m}$ modules of length $l_{b}$ (Fig. 3 ). We denote by $\mathscr{A}$ the beam axis and by $\mathscr{A}_{i}$ the part of the beam axis in the $i$ th module.

In the micro-model the kinematical state of the system is specified by means of the transverse displacement field

$$
u: \mathscr{A} \times \mathbb{R} \rightarrow \mathbb{R},
$$

and the set of $n_{m}$ nodal flux-linkages

$$
\psi_{h}: \mathbb{R} \rightarrow \mathbb{R}, \quad h=1, \ldots, n_{m} .
$$

In the homogenized model the kinematical state is specified by the macro-scalar fields

$$
\begin{aligned}
& U: \mathscr{A} \times \mathbb{R} \rightarrow \mathbb{R}, \\
& \Psi: \mathscr{A} \times \mathbb{R} \rightarrow \mathbb{R},
\end{aligned}
$$

representing the homogenized transverse displacement field and flux-linkage, respectively. At macro-level, the system is an electromechanical beam which, at each axial point $X$, has the microstructure shown in Fig. 3: the part $\mathscr{A}_{i}$ of the beam axis and the corresponding circuitry should be regarded as an infinitesimal neighbourhood of the generic point $X$. By locating the generic point in $\mathscr{A}_{i}$ by a micro-coordinate $\xi \in\left[-l_{b} / 2, l_{b} / 2\right]$, we assume that the micro-state of the neighbourhood $\mathscr{A}_{i}$ of the point $X$ is given as a function of the macro-kinematical fields through the following expansions:

$$
\begin{aligned}
& u(\xi, t)=U(X, t)+U^{\prime}(X, t) \xi+U^{\prime \prime}(X, t) \frac{\xi^{2}}{2}, \\
& \psi_{i \pm 1}(t)=\Psi(X, t) \pm \Psi^{\prime}(X, t) l_{b}+\Psi^{\prime \prime}(X, t) \frac{l_{b}^{2}}{2}, \\
& \psi_{i}(t)=\Psi(X, t) .
\end{aligned}
$$

The relations above can be regarded as a local (around the point $X$ ) kinematical map between the micro- and macro-models. This map assumes that $l_{b} / \lambda$ is sufficiently small. Here and henceforth, we denote by the upper-case letters the macro-quantities, by lower-case letters the micro-ones.

\subsection{Micro-model}

Once the micro-kinematical descriptors (3) and (4) are introduced, the micro-model of the system is obtained by specifying the internal and external virtual power functionals per module. We denote by $\dot{u}^{*}: \mathscr{A}_{i} \rightarrow \mathbb{R}$ and $\left\{\dot{\psi}_{h}^{*} \in \mathbb{R}\right\}$ the generalized virtual velocities acting as test functions and we assume the following expression for the internal $\left(\mathscr{P}_{\text {int }}(t)\right)$ and external $\left(\mathscr{P}_{\text {ext }}(t)\right)$ 
virtual powers:

$$
\begin{aligned}
& \mathscr{P}_{\text {int }}(t)=\chi_{i, 0}(t) \dot{\psi}_{i}^{*}+\chi_{i, 1}(t)\left(\dot{\psi}_{i+1}^{*}-\dot{\psi}_{i}^{*}\right)+\chi_{i, 2}(t)\left(\dot{\psi}_{i+1}^{*}-\dot{\psi}_{i-1}^{*}\right)+\int_{\mathscr{A}_{i}} m(\xi, t) \dot{u}^{*^{\prime \prime}}(\xi) \mathrm{d} \xi \\
& \mathscr{P}_{\text {ext }}(t)=\int_{\mathscr{A}_{i}} q(\xi, t) \dot{u}^{*}(\xi) \mathrm{d} \xi
\end{aligned}
$$

The first three terms of $\mathscr{P}_{\text {int }}(t)$ are electric contributions due to the virtual power spent by the currents $\left\{\chi_{i, 0}(t), \chi_{i, 1}(t), \chi_{i, 2}(t)\right\}$ on the potential differences $\left\{\dot{\psi}_{i}^{*}, \dot{\psi}_{i+1}^{*}-\dot{\psi}_{i}^{*}, \dot{\psi}_{i+1}^{*}-\dot{\psi}_{i-1}^{*}\right\}$ (refer to Fig. 3 for naming). The fourth term is the mechanical contribution due to the power spent by the bending moment $m(x, t)$ on the curvature velocity $\dot{u}^{* \prime \prime}(\xi)$. The bending moment and the electric currents are determined by the following micro-constitutive relations:

$$
\begin{aligned}
& m(\xi, t)=g_{m m} u^{\prime \prime}(\xi, t)+\dot{\psi}_{i}(t) \int_{\mathscr{A}_{i}} g_{m e}(\xi) \mathrm{d} \xi \\
& \chi_{i, 0}(t)=\mathscr{T}_{0}\left(\psi_{i}(t)\right)-\int_{\mathscr{A}_{i}} g_{m e}(\xi) \dot{u}^{\prime \prime}(\xi, t) \mathrm{d} \xi+\ddot{\psi}_{i}(t) \int_{\mathscr{A}_{i}} g_{e e}(\xi) \mathrm{d} \xi \\
& \chi_{i, 1}(t)=\mathscr{T}_{1}\left(\psi_{i+1}(t)-\psi_{i}(t)\right), \\
& \chi_{i, 2}(t)=\mathscr{T}_{2}\left(\psi_{i+1}(t)-\psi_{i-1}(t)\right)
\end{aligned}
$$

where

$$
\mathscr{T}_{h}(\cdot):=\frac{1}{r_{H}} \frac{\partial(\cdot)}{\partial t}+\frac{1}{L_{h}}(\cdot), \quad h=0,1,2
$$

express the elementary constitutive relations of the RL parallel impedances and $g_{m m}, g_{m e}$ and $g_{e e}$ are beam constitutive coefficients representing flexural stiffness, electromechanical coupling and electric capacitance per unit of line, respectively. When dynamic phenomena are considered, the basic inertia effects are modelled by accounting for a charge per unit of line given by

$$
q(\xi, t)=-\sigma(\xi) \ddot{u}(\xi, t) .
$$

The power functionals (7) and the constitutive equations (8a), (8b) and (10) assume an equivalent single layer Euler model for the layered piezoelectric beam and a constant distribution of the electric field inside each piezoelectric transducer. The specified beam model is deduced from a 3D Cauchy continuum description by assuming:

(i) uniaxial stress state both in the piezoelectric and elastic layers (i.e. the only non-vanishing stress component of the 3D Cauchy stress tensor is the axial normal stress),

(ii) perfect bonding conditions between the different layers and

(iii) purely extensional membranal behaviour of the piezoelectric layers.

In this way, the constitutive coefficients $\left(g_{m m}, g_{e e,} g_{m e}\right)$ appearing in Eq. (8) are expressed as a function of geometric and material properties of the 3D model as follows (see [22] for further details)

$$
g_{m m}(\xi)=Y_{b} w_{b} h_{b}^{3} / 12+2 Y_{p}^{E} w_{p} h_{p} h_{c}^{2} \Pi(\xi),
$$




$$
\begin{aligned}
& g_{m e}(\xi)=-2 d_{31} Y_{p}^{E} h_{c} w_{p} \Pi(\xi), \\
& g_{e e}(\xi)=2 \Pi(\xi) \varepsilon_{33}^{S} w_{p} / h_{p}, \\
& \sigma(\xi)=\rho_{b} w_{b} h_{b}+2 \rho_{p} w_{p} h_{p} \Pi(\xi) .
\end{aligned}
$$

In the expressions above, $Y_{b}$ is the Young modulus of the elastic layer, $Y_{p}^{E}$ the Young modulus of the piezoelectric layer for null electric field, $d_{31}$ the charge piezoelectric coupling coefficient, $\varepsilon_{33}^{S}$ the dielectric constant of the piezoelectric material for null mechanical deformation, $\rho_{b}$ and $\rho_{p}$ the mass densities of the elastic and piezoelectric layers, $h_{b}$ and $h_{p}$ their thicknesses, $w_{b}$ and $w_{p}$ the respective widths. Finally, $h_{c}=\left(h_{b}+h_{p}\right) / 2$ is the distance between the mid-planes of the piezoelectric and elastic layers. The function $\Pi(\xi)$ is defined to describe the discontinuities introduced by the piezoelectric transducers in the constitutive equations: its value is 1 in the axial region where the piezoelectric transducers are present, 0 everywhere else.

The following points deserve a comment:

- The presented model uses a completely linearized theory.

- In the electric constitutive equations (8b)-(8d) for the electric currents $\left\{\chi_{i, 0}(t), \chi_{i, 1}(t), \chi_{i, 2}(t)\right\}$, the terms given through $\mathscr{T}_{h}(\cdot)$ are the trivial constitutive equations for the two terminal networks consisting of a parallel connection of an inductor and a resistor. The terms dependent on $\ddot{\psi}_{i}(t)$ and $\dot{u}^{\prime \prime}(x, t)$ are related to the electric and coupling properties of the piezoelectric layers, respectively: the former is associated to the equivalent electric capacitance, the latter models the electromechanical coupling by an equivalent current source controlled by the beam deformation velocity. In the same fashion, in the mechanical constitutive equations (8a) we can distinguish between the standard purely mechanical contribution $g_{m m} u^{\prime \prime}(\xi, t)$ and the term due the piezoelectric coupling. The latter adds to the constitutive equation for the bending moment a contribution proportional to the potential difference $\dot{\psi}_{i}(t)$.

- The errors introduced by accounting barely for the extensional behaviour of the piezoelectric layers are acceptable if $h_{p} \ll h_{b}$.

- The second terms in expressions (11) for $g_{m m}(\xi)$ and $\sigma(\xi)$ model additional stiffness and mass per unit length due to the piezoelectric layers.

- The assumption of uniaxial stress state both in the piezoelectric and elastic layers, although standard, is a crude approximation of the actual stress distribution in the three-dimensional body. In particular, it is possible to show that it causes relevant errors in the estimation of the piezoelectric equivalent capacitance and, as a consequence (see the following sections), on the estimation of the values of the inductors and resistors which are required for an optimal vibration damping. In the present context, we keep this assumption in order to get qualitative results and simple symbolic expressions for the constitutive coefficients. However, the analysis of this problem deserves future investigation.

The interested reader can refer to [23-25] for generality about piezoelectric materials and to [26] for further details and references about models of laminated piezoelectric beams. 


\subsection{Macro-model}

Once the macro-micro-kinematical map (6) is prescribed, we define the power densities per unit length of the macro-model as the average of the corresponding micro-quantities over a module. Hence, by introducing Eq. (6) into Eq. (7), the following expressions for the internal and external power densities are found:

$$
\begin{aligned}
& \overline{\mathscr{P}}_{\text {int }}(X, t)=M(X, t) \dot{U}^{*^{\prime \prime}}(X)+\Upsilon_{0}(X, t) \dot{\Psi}^{*}(X)+\Upsilon_{1}(X, t) \dot{\Psi}^{*^{\prime}}(X)+\Upsilon_{2}(X, t) \dot{\Psi}^{*^{\prime \prime}}(X), \\
& \overline{\mathscr{P}}_{\text {ext }}(X, t)=B(X, t) \dot{U}^{*}(X)+B_{1}(X, t) \dot{U}^{*^{\prime}}(X)+B_{2}(X, t) \dot{U}^{*^{\prime \prime}}(X)
\end{aligned}
$$

and the following macro-constitutive relations are derived from Eqs. (8) and (10):

$$
\begin{aligned}
& M(X, t)=\bar{g}_{m m} U^{\prime \prime}(X, t)+\bar{g}_{m e} \dot{\Psi}(X, t), \\
& B(X, t)=-\bar{\sigma} \ddot{U}(X, t), \\
& \Upsilon_{0}(X, t)=\frac{1}{l_{b}} \mathscr{T}_{0}(\Psi(X, t))+\bar{g}_{e e} \ddot{\Psi}(X, t)-\bar{g}_{m e} \dot{U}^{\prime \prime}(X, t), \\
& \Upsilon_{1}(X, t)=l_{b}\left(\mathscr{T}_{1}+4 \mathscr{T}_{2}\right)\left(\Psi^{\prime}(X, t)\right)+\frac{l_{b}^{2}}{2} \mathscr{T}_{1}\left(\Psi^{\prime \prime}(X, t)\right), \\
& \Upsilon_{2}(X, t)=\frac{l_{b}^{2}}{2} \mathscr{T}_{1}\left(\Psi^{\prime}(X, t)\right)+\frac{l_{b}^{3}}{4} \mathscr{T}_{1}\left(\Psi^{\prime \prime}(X, t)\right),
\end{aligned}
$$

where

$$
\begin{aligned}
& \bar{g}_{m m}=\frac{1}{l_{b}} \int_{\mathscr{A}_{i}} g_{m m}(\xi) \mathrm{d} \xi=\frac{Y_{b} w_{b} h_{p}^{3}}{12}+2 \vartheta Y_{p}^{E} h_{c}^{2} h_{p} w_{p}, \\
& \bar{g}_{m e}=\frac{1}{l_{b}} \int_{\mathscr{A}_{i}} g_{m e}(\xi) \mathrm{d} \xi=-2 \vartheta d_{31} Y_{p}^{E} h_{c} w_{p}, \\
& \bar{g}_{e e}=\frac{1}{l_{b}} \int_{\mathscr{A}_{i}} g_{e e}(\xi) \mathrm{d} \xi=2 \vartheta \varepsilon_{33}^{S} w_{p} / h_{p}, \\
& \bar{\sigma}=\frac{1}{l_{b}} \int_{\mathscr{A}_{i}} \sigma(\xi) \mathrm{d} \xi=\rho_{b} w_{b} h_{b}+2 \vartheta \rho_{p} w_{p} h_{p} .
\end{aligned}
$$

The longitudinal covering factor $\vartheta:=h_{p} / h_{b}$ is used. It is defined as the ratio between the length $l_{p}$ of the piezoelectric transducers and the length $l_{b}$ of a beam element. The first term in $\overline{\mathscr{P}}_{\text {int }}(X, t)$ represents the mechanical power density due to an homogenized curvature virtual velocity $\dot{U}^{*^{\prime \prime}}(X)$. The other terms are electrical contributions in which $\left\{\dot{\Psi}^{*}(X), \dot{\Psi}^{*^{\prime}}(X), \dot{\Psi}^{*^{\prime \prime}}(X)\right\}$ are generalized potential differences and $\left\{\Upsilon_{0}(X, t), \Upsilon_{1}(X, t), \Upsilon_{2}(X, t)\right\}$ are generalized currents appearing in the homogenized model of the electromechanical system. The external power density $\mathscr{\mathscr { P }}_{\text {ext }}(X, t)$ accounts for the macro-inertia effects arising from those at the micro-level through the kinematical map (6). In what follows, the higher-order inertial forces $B_{1}(X, t)$ and $B_{2}(X, t)$, which are related to the rotation and curvature velocities of each beam module, are neglected by assuming that $l_{b} / \lambda$ is sufficiently small. 
Finally, a weak form of the balance equations in the macro-model is given by imposing that the following power balance holds for all the admissible test functions $\left\{\dot{U}^{*}, \dot{\Psi}^{*}\right\}$ :

$$
\begin{aligned}
& \int_{\mathscr{A}}\left(M(X, t) \dot{U}^{*^{\prime \prime}}(X)+\Upsilon_{0}(X, t) \dot{\Psi}^{*}(X)+\Upsilon_{1}(X, t) \dot{\Psi}^{*^{\prime}}(X)+\Upsilon_{2}(X, t) \dot{\Psi}^{*^{\prime \prime}}(X)\right) \mathrm{d} X \\
& =\int_{\mathscr{A}} B(X, t) \dot{U}^{*}(X) \mathrm{d} X .
\end{aligned}
$$

The corresponding strong-form balance equations in $\mathscr{A}$ are

$$
\begin{aligned}
& M^{\prime \prime}(X, t)-B(X, t)=0, \\
& \Upsilon_{2}{ }^{\prime \prime}(X, t)-\Upsilon_{1}{ }^{\prime}(X, t)+\Upsilon_{0}(X, t)=0 .
\end{aligned}
$$

Only the boundary conditions verifying the following relations for each admissible kinematical field are consistent with the power balance (15):

$$
\begin{aligned}
& {\left[M(X, t) \dot{U}^{*^{\prime}}(X)-M^{\prime}(X, t) \dot{U}(X)\right]_{\partial \mathscr{A}}=0,} \\
& {\left[\Upsilon_{2}(X, t) \dot{\Psi}^{*^{\prime}}(X)-\Upsilon_{2}^{\prime}(X, t) \dot{\Psi}^{*}(X)+\Upsilon_{1}(X, t) \dot{\Psi}^{*}(X)\right]_{\partial \mathscr{A}}=0 .}
\end{aligned}
$$

The substitution of the macro-constitutive equations (13) into the balance equations (16) allows for the derivation of the following homogenized equations of motion for the PEM beam:

$$
\begin{aligned}
& \bar{g}_{m m} U^{\mathrm{IV}}+\bar{g}_{m e} \dot{\Psi}^{\prime \prime}+\bar{\sigma} \ddot{U}=0, \\
& \frac{l_{b}^{3}}{4} \mathscr{T}_{1}\left(\Psi^{\mathrm{IV}}\right)-l_{b}\left(\mathscr{T}_{1}+4 \mathscr{T}_{2}\right)\left(\Psi^{\prime \prime}\right)+\frac{1}{l_{b}} \mathscr{T}_{0}(\Psi)+\bar{g}_{e e} \ddot{\Psi}-\bar{g}_{m e} \dot{U}^{\prime \prime}=0 .
\end{aligned}
$$

It is convenient to write down the expanded version of system (18) in non-dimensional matrix form by introducing the state vector

$$
\mathbf{s}(X, t)=\{U(X, t), \Psi(X, t)\}^{t},
$$

in which the non-dimensional mechanical and electrical fields are collected (the dimensionless variables are denoted with the same letters of the dimensional ones). System (18) is rewritten as follows:

$$
\ddot{\mathbf{s}}(X, t)+\mathscr{D}^{(1)} \dot{\mathbf{s}}(X, t)+\mathscr{D}^{(0)} \mathbf{s}(X, t)=0,
$$

where $\mathscr{D}_{1}$ and $\mathscr{D}_{0}$ are space-differential operators. If the following scaling time and electric fluxlinkage are chosen

$$
t_{0}=X_{0}^{2} \sqrt{\frac{\bar{\rho}}{\bar{g}_{m m}}}, \quad \Psi_{0}=\sqrt{\frac{\bar{\rho}}{\bar{g}_{e e}}} U_{0}
$$

then,

$$
\mathscr{D}^{(1)}=\left[\begin{array}{cc}
0 & \gamma \frac{\partial^{2}}{\partial X^{2}} \\
-\gamma \frac{\partial^{2}}{\partial X^{2}} & \delta_{0}-\delta_{2} \frac{\partial^{2}}{\partial X^{2}}+\delta_{4} \frac{\partial^{4}}{\partial X^{4}}
\end{array}\right], \quad \mathscr{D}^{(0)}=\left[\begin{array}{cc}
\frac{\partial^{4}}{\partial X^{4}} & 0 \\
0 & \beta_{0}-\beta_{2} \frac{\partial^{2}}{\partial X^{2}}+\beta_{4} \frac{\partial^{4}}{\partial X^{4}}
\end{array}\right],
$$


where the following sets of non-dimensional tuning $\left(\beta_{0}, \beta_{2}, \beta_{4}\right)$ and damping $\left(\delta_{0}, \delta_{2}, \delta_{4}\right)$ parameters are defined

$$
\begin{aligned}
& \beta_{0}=\frac{1}{L_{0}} n c_{\beta}, \quad \beta_{2}=\left(\frac{1}{L_{1}}+\frac{4}{L_{2}}\right) \frac{c_{\beta}}{n}, \quad \beta_{4}=\frac{1}{4 L_{1}} \frac{c_{\beta}}{n^{3}}, \\
& \delta_{0}=\frac{1}{R_{0}} n c_{\delta}, \quad \delta_{2}=\left(\frac{1}{R_{1}}+\frac{4}{R_{2}}\right) \frac{c_{\delta}}{n}, \quad \delta_{4}=\frac{1}{4 R_{1}} \frac{c_{\delta}}{n^{3}}
\end{aligned}
$$

with

$$
c_{\beta}=\frac{\bar{\sigma}}{\bar{g}_{e e} \bar{g}_{m m}} X_{0}^{3}, \quad c_{\delta}=\frac{1}{\bar{g}_{e e}} \sqrt{\frac{\bar{\sigma}}{\bar{g}_{m m}}} X_{0} .
$$

The coupling parameter $\gamma$ is given by

$$
\gamma=\frac{\bar{g}_{m e}}{\sqrt{\bar{g}_{m m} \bar{g}_{e e}}} \text {. }
$$

The number of modules $n$ in a characteristic length $X_{0}$ is introduced as

$$
n=\frac{X_{0}}{l_{b}}
$$

Thus, once the mechanical properties are fixed, all the parameters (23) are expressed as a function of the number of modules per unit length $n$, the inductances $\left(L_{0}, L_{1}, L_{2}\right)$, and the resistances $\left(R_{0}, R_{1}, R_{2}\right)$ (see Fig. 3). The scaling variables (21) were chosen in order to impose equal to the unity the coefficient of $U^{\mathrm{IV}}$ in the mechanical equation and to maintain the skew symmetry in the electromechanical (gyroscopic-type) coupling.

The equations of motion (20) refer to the circuit topology described in Fig. 2. We report in Table 1 how the circuit parameters $\left(R_{0}, R_{1}, R_{2}, L_{0}, L_{1}, L_{2}\right)$ can be chosen in order to synthesize PEM beams in which the electric evolution is governed (as far as a homogenized model is concerned) by each one of the differential equations (2). It this way we indirectly show also that every PDE in list (2) can be recognized as the evolution equation for the corresponding circuit in Fig. 5 (an infinite value for an electric inductance or resistance is equivalent to an open circuit condition between the corresponding terminals). We explicitly remark that, when the beam is coupled with one of the distributed electric vibration absorbers (2), only one among the three

Table 1

Electric parameters in order to obtain the five distributed vibration absorbers from the generic network topology

\begin{tabular}{llllll}
\hline Network & $(\mathrm{Z}, \mathrm{Z})$ & $(\mathrm{S}, \mathrm{Z})$ & $(\mathrm{S}, \mathrm{S})$ & $(\mathrm{F}, \mathrm{Z})$ & $(\mathrm{F}, \mathrm{S})$ \\
\hline$L_{0}$ & $L$ & $\infty$ & $\infty$ & $\infty$ & $\infty$ \\
$L_{1}$ & $\infty$ & $\infty$ & $\infty$ & $L$ & $L$ \\
$L_{2}$ & $\infty$ & $L$ & $L$ & $-4 L$ & $-4 L$ \\
$R_{0}$ & $R$ & $R$ & $\infty$ & $R$ & $\infty$ \\
$R_{1}$ & $\infty$ & $\infty$ & $\infty$ & $\infty$ & $\infty$ \\
$R_{2}$ & $\infty$ & $\infty$ & $R$ & $\infty$ & $R$ \\
\hline
\end{tabular}




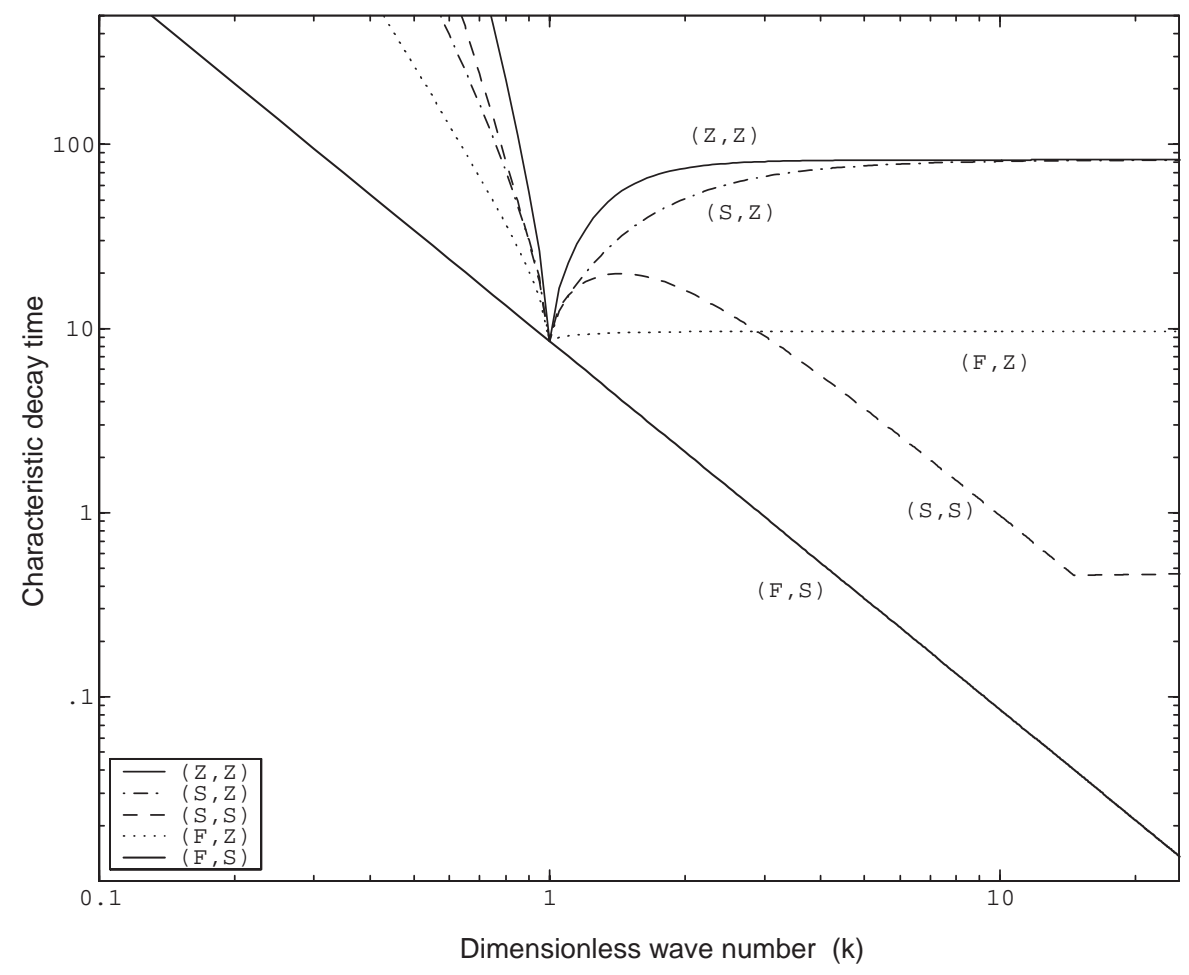

Fig. 5. Characteristic decay time function $T_{\mathrm{opt}}(k, 1)$ for the five network configurations.

Table 2

Non-vanishing tuning and damping parameters for the five distributed vibration absorbers

\begin{tabular}{llllll}
\hline Network & $(\mathrm{Z}, \mathrm{Z})$ & $(\mathrm{S}, \mathrm{Z})$ & $(\mathrm{S}, \mathrm{S})$ & $(\mathrm{F}, \mathrm{Z})$ & $(\mathrm{F}, \mathrm{S})$ \\
\hline Tuning & $\beta_{0}$ & $\beta_{2}$ & $\beta_{2}$ & $\beta_{4}$ & $\beta_{4}$ \\
Damping & $\delta_{0}$ & $\delta_{0}$ & $\delta_{2}$ & $\delta_{0}$ & $\delta_{2}$ \\
\hline
\end{tabular}

tuning parameters $\left(\beta_{0}, \beta_{2}, \beta_{4}\right)$ and one among the three damping parameters $\left(\delta_{0}, \delta_{2}, \delta_{4}\right)$ is not vanishing, as it is ruled by Table 2 .

\section{Electromechanical dynamics}

In the previous section, the evolution equations for homogenized models of the PEM beams were derived. In the present section, the dependence of their dynamic properties on the electric parameters is analysed. To this end, the temporal decay of $k$-waves (i.e. solutions in the form of electromechanical waves characterized by a single real wave number $k$ ) evolving in infinite PEM beams is studied. For the five absorbers, the optimal electric parameters to damp a $k$-wave are 
found and the corresponding damping performances are compared. An application to the case of a simply supported beam is presented.

\section{1. $k$-waves solutions}

The evolution equations of the five PEM beams are in form (20), where $\mathbf{s}(X, t)$ is the vectorvalued function describing the local, instantaneous electromechanical state of the system. Let us look for the temporal evolution of solutions $\mathrm{s}_{k}(X, t)$ in the form

$$
\mathbf{s}_{k}(X, t)=\mathbf{s}(t) \mathrm{e}^{i k X},
$$

where in $\mathrm{s}(t)$ only the temporal dependence is left. We call a solution of this form a $k$-wave, which is a particular standing wave as defined by Courant and Hilbert [28]. The analysis of $k$-waves is relevant because, by means of a Fourier transform (or series), the general solution to a dynamic problem for a infinite (or finite) beam can be written as the superposition of standing waves, each one with a fixed wave number. We do not reserve any physical meaning to the term standing wave, and it is simply used to denote solutions in the form of (27).

The substitution of the waveform solution (27) into the equations of motion (20) leads to the following system of two second-order ordinary differential equations for the temporal evolution $\mathrm{s}(t)$ of a $k$-wave:

$$
\ddot{\mathrm{s}}(t)+D_{k}^{(1)} \dot{\mathbf{s}}(t)+D_{k}^{(0)} \mathbf{s}(t)=0,
$$

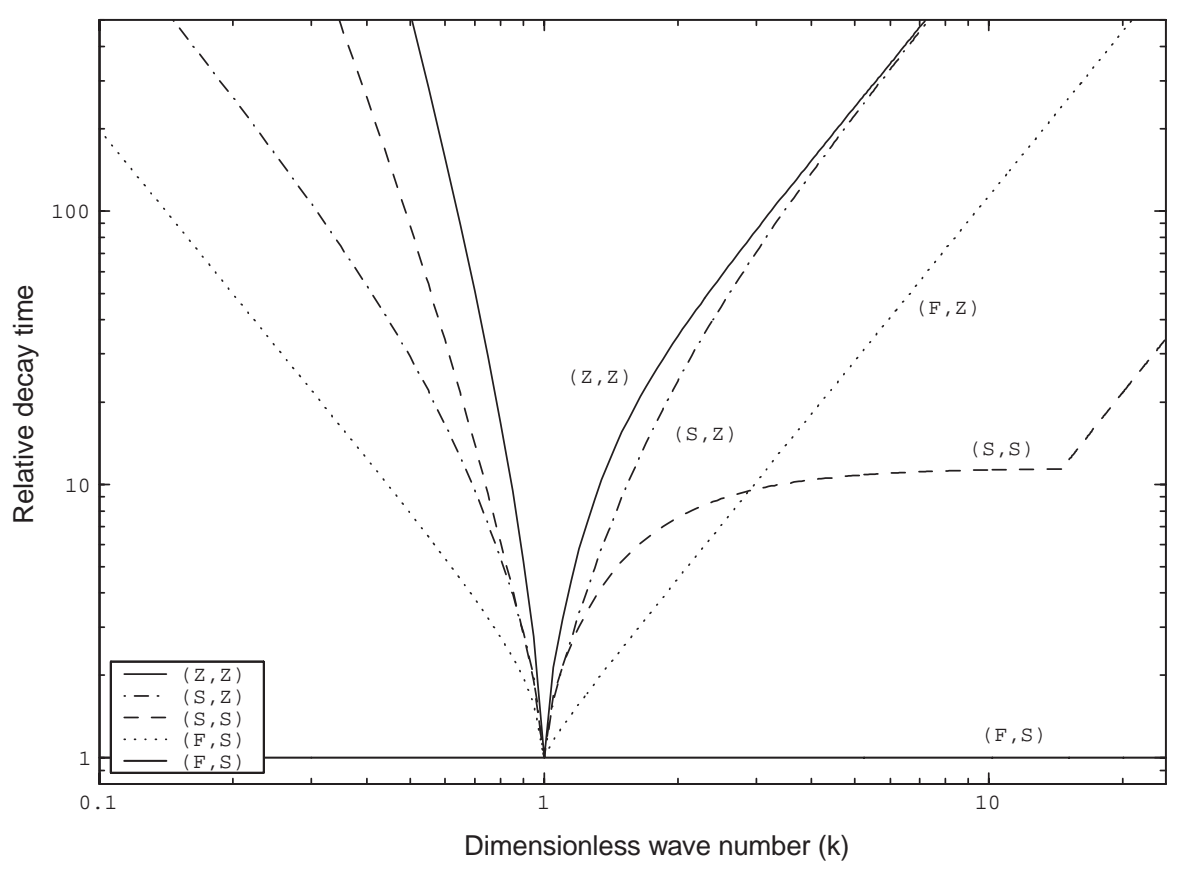

Fig. 6. Relative decay time $\tilde{T}_{\text {opt }}(k, 1)$ for the five network configurations. 
where $D_{k}^{(1)}, D_{k}^{(0)}$ are real algebraic operators since only even order spatial derivatives appear in $\mathscr{D}_{k}^{(1)}$ and $\mathscr{D}_{k}^{(0)}$. For the general system in Fig. 2

$$
D_{k}^{(0)}=\left[\begin{array}{cc}
k^{4} & 0 \\
0 & \beta_{0}+\beta_{2} k^{2}+\beta_{4} k^{4}
\end{array}\right], \quad D_{k}^{(1)}=\left[\begin{array}{cc}
0 & -\gamma k^{2} \\
\gamma k^{2} & \delta_{0}+\delta_{2} k^{2}+\delta_{4} k^{4}
\end{array}\right] .
$$

System (28) can be conveniently rewritten as a system of four first-order ordinary differential equations with constant coefficients, as follows:

$$
\dot{\mathbf{y}}(t)=\mathbf{A}_{k} \mathbf{y}(t)
$$

with

$$
\mathbf{y}(t)=\left\{\begin{array}{c}
\mathbf{s}(t) \\
\dot{\mathbf{s}}(t)
\end{array}\right\}, \quad \mathbf{A}_{k}=\left[\begin{array}{cc}
0_{2} & I_{2} \\
-D_{k}^{(0)} & -D_{k}^{(1)}
\end{array}\right],
$$

where $0_{2}, I_{2}$ denote the zero and identity $2 \times 2$ matrices, respectively. The subscript $k$ on $\mathbf{A}_{k}$ underlines that system (30) is written for the temporal evolution of $k$-waves. For the system in Fig. 2:

$$
\mathbf{A}_{k}=\left[\begin{array}{cccc}
0 & 0 & 1 & 0 \\
0 & 0 & 0 & 1 \\
-k^{4} & 0 & 0 & \gamma k^{2} \\
0 & -\beta(k) & -\gamma k^{2} & -\delta(k)
\end{array}\right],
$$

where $\beta(k)$ and $\delta(k)$ are defined by

$$
\begin{aligned}
& \beta(k):=\beta_{0}+k^{2} \beta_{2}+k^{4} \beta_{4}, \\
& \delta(k):=\delta_{0}+k^{2} \delta_{2}+k^{4} \delta_{4}
\end{aligned}
$$

and they can be regarded as equivalent tuning and damping parameters, respectively. The characteristic polynomial of $\mathbf{A}_{k}$ is

$$
\mu^{4}+\delta(k) \mu^{3}+\left(\beta(k)+k^{4}\left(1+\gamma^{2}\right)\right) \mu^{2}+k^{4} \delta(k) \mu+k^{4} \beta(k)=0 .
$$

The solution of Eq. (30), starting from a generic initial condition

$$
\mathbf{y}_{0}=\left\{\begin{array}{c}
\mathrm{s}(0) \\
\dot{\mathrm{s}}(0)
\end{array}\right\}
$$

is given by

$$
\mathbf{y}(t)=\mathbf{e}^{\mathbf{A}_{k} t} \mathbf{y}_{0} .
$$

In order to discuss the qualitative properties of $k$-waves of form (27), we give a deep insight into Eq. (36) by recalling the fundamental features of the solution of a linear system of autonomous differential equations and discussing how they are related to the spectrum $\sigma$ of $\mathbf{A}_{k}$.

In general, the spectrum $\sigma$, collecting the eigenvalues of the real operator $\mathbf{A}_{k}$ is composed of $p$ real eigenvalues $\left\{\mu_{1}, \ldots, \mu_{p}\right\}$ and $q$ complex conjugate pairs $\left\{\mu_{p+1}, \mu_{p+1}^{*}, \ldots, \mu_{p+q}, \mu_{p+q}^{*}\right\}$, with $p+$ $2 q=4$. It can be shown that, since we are dealing with dissipative systems, all the eigenvalues must have a non-positive real part. Let us associate to each real eigenvalue $\mu_{m}$ and to each 
complex pair $\left\{\mu_{n}, \mu_{n}^{*}\right\}$ the real generalized eigenspaces $\mathscr{N}_{m}, \mathscr{N}_{n}$, respectively (the real eigenspace $\mathscr{N}_{n}$ associated to the complex conjugate pair $\left\{\mu_{n}, \mu_{n}^{*}\right\}$ can be defined as the vectorial space generated by the set of vector $\left\{\operatorname{Re}\left(\mathbf{e}_{1}^{n}\right),-\operatorname{Im}\left(\mathbf{e}_{1}^{n}\right), \ldots, \operatorname{Re}\left(\mathbf{e}_{k}^{n}\right),-\operatorname{Im}\left(\mathbf{e}_{k}^{n}\right)\right\}$, where $\left\{\mathbf{e}_{1}^{n}, \ldots, \mathbf{e}_{k}^{n}\right\}$ are the (complex) generalized eigenvectors associated to $\mu_{n}$ ). Hence, let us recall the following properties (see [27] for complete proofs). We denote by $\mathscr{V}$ the (four-dimensional) vector space in which y lies:

(1) The vector space $\mathscr{V}$ can be decomposed as direct sum of the generalized eigenspaces

$$
\left\{\mathscr{N}_{1}, \ldots, \mathscr{N}_{p}, \mathscr{N}_{p+1}, \ldots, \mathscr{N}_{p+q}\right\}
$$

(2) Solution (36) can be decomposed into

$$
\mathbf{y}(t)=\sum_{j=1}^{p+q} \mathbf{y}^{(j)}(t)
$$

where each $\mathbf{y}^{(j)}(t)$ has values in $\mathscr{N}_{j}$ and it is the (unique) solution starting from the initial data $\mathbf{y}_{0}^{(j)}$, projection of $\mathbf{y}_{0}$ on the corresponding generalized eigenspace $\mathscr{N}_{j}$.

(3) For each $j$ there exists an $l_{j} \geqslant 0$ such that $\mathbf{y}^{(j)}(t)$ is a linear combination of terms of the form

$$
t^{l} \mathrm{e}^{\operatorname{Re}\left(\mu_{j}\right) t}
$$

for $j=1, \ldots, p$ and of the form

$$
t^{l} \mathrm{e}^{\operatorname{Re}\left(\mu_{j}\right) t} \sin \left(\operatorname{Im}\left(\mu_{j}\right)\right), t^{l} \mathrm{e}^{\operatorname{Re}\left(\mu_{j}\right) t} \cos \left(\operatorname{Im}\left(\mu_{j}\right)\right)
$$

for $j=p+1, \ldots, p+q$, where in general $0 \leqslant l \leqslant l_{j} \leqslant \operatorname{dim}\left(\mathscr{N}_{j}\right)$ and $l_{j}=0$ if and only if $\mathscr{N}_{j} \equiv$ $\operatorname{ker}\left(\mathbf{A}_{k}-\mu_{j} \mathbf{I}\right)$.

It is easy now to show that, for each $j=1, \ldots, p+q$, for each $\mathbf{y}_{0}$ a constant $M_{j}\left(\mathbf{y}_{0}\right) \geqslant 0$ exists such that, for each $t>0$,

$$
\mathbf{y}^{(j)}(t) \leqslant M_{j} \mathrm{e}^{-\varrho_{j} t},
$$

where

$$
\varrho_{j}:=-\operatorname{Re}\left(\mu_{j}\right) .
$$

Hence, the following important properties are verified for the temporal evolution $\mathbf{s}(t)$ of a standing wave with a wave number $k>0$ :

(1) For each $j=1, \ldots, p+q$, the projection of $\mathrm{s}(t)$ in the eigenspace $\mathscr{N}_{j}$ can be characterized by the exponential decay rate $\varrho_{j}$ defined by relationship (42). Equivalently, we can define the characteristic damping time as $\tau_{j}:=1 / \varrho_{j}$. For complex conjugate eigenvalues, we can introduce also a damping ratio

$$
\zeta_{i}:=-\frac{\operatorname{Re}\left(\mu_{j}\right)}{\sqrt{\operatorname{Re}\left(\mu_{j}\right)^{2}+\operatorname{Im}\left(\mu_{j}\right)^{2}}} .
$$


(2) The whole solution $\mathrm{s}(t)$ can be characterized by an exponential decay rate

$$
\varrho:=\min _{j=1, \ldots, p+q}\left(-\operatorname{Re}\left(\mu_{j}\right)\right)
$$

and by a damping time

$$
\tau:=\max _{j=1, \ldots, p+q}\left(\tau_{j}\right)=1 / \varrho .
$$

Moreover, it is possible to define the following system damping ratio:

$$
\zeta:=\min _{j=1, \ldots, p+q}\left(\zeta_{j}\right) .
$$

All the quantities above refer to a fixed wave number $k$ and, when necessary, the explicit functional dependence on $k$ is underlined. The proposed characterization for the temporal evolution $\mathbf{s}(t)$ is based only on the system eigenvalues and is completely independent of the initial data $\mathbf{y}_{0}$.

\subsection{Optimal damping of $k$-waves}

The optimization and performance estimation of the five distributed vibration absorbers which are characterized by the equations of motion (2a)-(2e) is subdivided into three steps: (i) definition of a performance index (or a cost function) which is related to the damping properties of the system; (ii) maximization of the performance index for a single wave number $\bar{k}$ in order to obtain the optimal design of the electric passive controller (the so obtained vibration absorbers are called $\bar{k}$-absorbers); and (iii) analysis and comparison of the damping performances of the five $\bar{k}$-absorbers for wave numbers different from $\bar{k}$.

The distributed array of piezoelectric transducers and the interconnecting electric network were conceived to obtain a structural modification of a beam aimed at passive vibration control. We do not attempt to find any optimal interconnecting topology, but, for a single wave number, we state a general optimization problem for the system in Fig. 2, in order to find the optimal equivalent tuning and damping parameters $\beta(k)$ and $\delta(k)$ defined by Eqs. (33). The optimal tuning and damping parameters for each one of the five circuit topologies in Fig. 4 are found as particular cases since, for each topology, only one $\beta$ among the three tuning parameters $\left(\beta_{0}, \beta_{2}, \beta_{4}\right)$ and one $\delta$ among the three damping parameters $\left(\delta_{0}, \delta_{2}, \delta_{4}\right)$ is not vanishing, as shown in Table 2.

\subsubsection{Performance index}

We optimize the system performance by minimizing the temporal decay obtained for a standing electromechanical wave in form (27) with a fixed wave number $\vec{k}$. Since the temporal evolution of a standing wave is determined by the system of the two second-order differential equations (28), we can note that, once the wave number is fixed to $\bar{k}$, the optimal design of the electric part of that system is equivalent to the optimization of a dissipative one-degree-of-freedom dynamic vibration absorber gyroscopically coupled to a harmonic oscillator. Hence, the optimization techniques which were developed for that application can be fruitfully revisited. In particular, we follow a pole-placement method analog to the one that was adopted by Hagood and Von Flotow [2] for the optimization of a resonant shunted piezoelectric transducer. 
We choose as performance index $P I$ the system decay rate which was introduced by definition (44). Let us underline its functional dependence on the wave number $k$, the tuning parameter $\beta$ and damping parameter $\delta$ by writing

$$
P I(k, \beta, \delta):=\varrho(k, \beta, \delta)=\min _{j=1, \ldots, 4}\left(-\operatorname{Re}\left(\mu_{j}(k, \beta, \delta)\right)\right) .
$$

We assume that $\delta, \beta \geqslant 0$. Definition (47) implies the assumption of a pole-placement criterion for the system optimization. Indeed, it completely characterizes the system performance by the location of the eigenvalues on the complex plane. The optimization of a given network implies the maximization of index (47) with respect to $(\beta, \delta)$ when the wave number $k$ is fixed and equal to $\bar{k}$.

\subsubsection{Optimization}

Finding analytical expressions for the parameters $\beta$ and $\delta$ which maximize the performance index (47) is not a trivial task. Indeed, the evaluation of $P I$ requires the determination of the roots of the fourth-order characteristic polynomial (34). Although analytical formulae are available, they are cumbersome and do not allow a easy quantitative analysis. On the other hand, a numerical optimization of index (47) with respect to $\beta$ and $\delta$ for a single wave number is always possible, but it does not allow for an analytical definition of the relations expressing the optimal parameters. We proceed by employing a useful property of index (47): it is maximum when the four eigenvalues of $\mathbf{A}_{k}$ appear in the form of two coincident complex conjugate pairs. This property is widely exploited in the literature (see e.g. [2,4,9], where the optimization of dynamic vibration absorbers is carried out by means of a pole-placement method). The characteristic polynomial of the system governing the temporal evolution of a $\bar{k}$-wave is given by expression (34) for $k \rightarrow \vec{k}$. A fourth-order polynomial $\bar{p}(\mu)$, the roots of which are two coincident complex conjugate pairs, has the form

$$
\bar{p}(\mu)=(\mu-(a+i b))^{2}(\mu-(a-i b))^{2},
$$

where $a$ and $b$ are real numbers specifying the location of the roots. In order to impose that $p(\mu)=\bar{p}(\mu)$ for each $\mu$, the following non-linear system in $\left(a, b, \delta^{\text {opt }}, \beta^{\text {opt }}\right)$ must be satisfied:

$$
\begin{aligned}
& \delta^{\mathrm{opt}}(\bar{k})=-4 a, \\
& \beta^{\mathrm{opt}}(\bar{k})+\bar{k}^{4}+\bar{k}^{4} \gamma^{2}=\left(6 a^{2}+2 b^{2}\right), \\
& \bar{k}^{4} \delta^{\mathrm{opt}}(\bar{k})=-4 a\left(a^{2}+b^{2}\right), \\
& \bar{k}^{4} \beta^{\mathrm{opt}}(\bar{k})=\left(a^{2}+b^{2}\right)^{2} .
\end{aligned}
$$

It is possible to show that, for $\gamma<2$, the only admissible $(\beta, \delta>0$ and $a, b \in \mathbb{R})$ solution of (49) furnishes the optimal equivalent parameters

$$
\beta^{\mathrm{opt}}(\bar{k})=\bar{k}^{4}, \quad \delta^{\mathrm{opt}}(\bar{k})=2 \gamma \bar{k}^{2} .
$$

The corresponding location of the eigenvalues is expressed by

$$
a=-\frac{1}{2} \bar{k}^{2} \gamma, \quad b=\bar{k}^{2} \sqrt{1-\frac{\gamma^{2}}{4}} .
$$


The associated damping ratio is

$$
\zeta=-\frac{a}{\sqrt{a^{2}+b^{2}}}=\frac{\gamma}{2} .
$$

This optimal damping ratio depends only on the non-dimensional coupling parameter $\gamma$ and it is the same for the five network configurations.

By recalling definitions (33) for $\beta(k)$ and $\delta(k)$ and that, for each network configuration only one among $\left(\beta_{0}, \beta_{2}, \beta_{4}\right)$ and one among $\left(\delta_{0}, \delta_{2}, \delta_{4}\right)$ is not vanishing, the optimal tuning and damping parameters $\left(\beta_{i}, \delta_{j}\right)$ of the $i$ th order network with a $j$ th order dissipation can be found from expressions (50) of the optimal equivalent parameters $\left(\beta^{\mathrm{opt}}, \delta^{\mathrm{opt}}\right)$. The corresponding expressions are reported in Table 3. The optimal inductors and resistors as a function of the number of modules in a characteristic length $n$ and of the design value of the wave number $\bar{k}$ are immediately found through definitions (23). The corresponding expressions are reported in Table 4. Looking at their expressions for the different circuit topologies, we can note the following points:

(1) Dependence on location: The optimal values of the resistors and the inductors in each module are the same if they are located in the same circuit branch (e.g. the optimal value of the ground resistor $R_{0}(\bar{k})$ is the same in the $(\mathrm{Z}, \mathrm{Z}),(\mathrm{S}, \mathrm{Z})$ and $(\mathrm{F}, \mathrm{Z})$ networks, and the optimal value of the line inductor $L_{2}(\bar{k})$ is the same in the (S, Z) and (S, S) networks).

(2) Dependence on wave number: If the resistors and the inductors are properly located, their optimal values are independent of the wave number $\bar{k}$. Indeed, the optimal resistors are independent of the wave number if the corresponding dissipation term appears in Eqs. (20) with a second-order spatial derivative ((S,S) and (F,S) networks); the optimal inductors are independent of the wave number if they are located so as to lead to fourth-order networks $((F, Z)$ and $(F, S))$.

Table 3

Optimal tuning and damping parameters for the five distributed vibration absorbers

\begin{tabular}{llllll}
\hline Network & $(\mathrm{Z}, \mathrm{Z})$ & $(\mathrm{S}, \mathrm{Z})$ & $(\mathrm{S}, \mathrm{S})$ & $(\mathrm{F}, \mathrm{Z})$ & $(\mathrm{F}, \mathrm{S})$ \\
\hline Optimal tuning & $\beta_{0}^{\mathrm{opt}}(\bar{k})=\bar{k}^{4}$ & $\beta_{2}^{\mathrm{opt}}(\bar{k})=\bar{k}^{2}$ & $\beta_{2}^{\mathrm{opt}}(\bar{k})=\bar{k}^{2}$ & $\beta_{4}^{\mathrm{opt}}(\bar{k})=1$ & $\beta_{4}^{\mathrm{opt}}(\bar{k})=1$ \\
Optimal damping & $\delta_{0}^{\mathrm{opt}}(\bar{k})=2 \gamma \bar{k}^{2}$ & $\delta_{0}^{\mathrm{opt}}(\bar{k})=2 \gamma \bar{k}^{2}$ & $\delta_{2}^{\mathrm{opt}}(\bar{k})=2 \gamma$ & $\delta_{0}^{\mathrm{opt}}(\bar{k})=2 \gamma \bar{k}^{2}$ & $\delta_{2}^{\mathrm{opt}}(\bar{k})=2 \gamma$ \\
\hline
\end{tabular}

Table 4

Optimal inductors and resistors in each module for the five distributed vibration absorbers

\begin{tabular}{lll}
\hline Network & Optimal inductors & Optimal resistors \\
\hline$(\mathrm{Z}, \mathrm{Z})$ & $L_{0}^{\mathrm{opt}}(\bar{k})=\frac{c_{\beta}}{\bar{k}^{4}} n$ & $R_{0}^{\mathrm{opt}}(\bar{k})=\frac{c_{\delta}}{2 \gamma \bar{k}^{2}} n$ \\
$(\mathrm{~S}, \mathrm{Z})$ & $R_{0}^{\mathrm{opt}}(\bar{k})=\frac{c_{\delta}}{2 \gamma \bar{k}^{2}} n$ \\
$(\mathrm{~S}, \mathrm{~S})$ & $L_{2}^{\mathrm{opt}}(\bar{k})=\frac{4 c_{\beta}}{\bar{k}^{2}} \frac{1}{n}$ & $R_{2}^{\mathrm{opt}}(\bar{k})=\frac{2 c_{\delta}}{\gamma} \frac{1}{n}$ \\
$(\mathrm{~F}, \mathrm{Z})$ & $L_{2}^{\mathrm{opt}}(\bar{k})=\frac{4 \beta_{1}}{\bar{k}^{2}} \frac{1}{n}$ & $R_{0}^{\mathrm{opt}}(\bar{k})=\frac{c_{\delta}}{2 \gamma \bar{k}^{2}} n$ \\
$(\mathrm{~F}, \mathrm{~S})$ & $L_{1}^{\mathrm{opt}}(\bar{k})=\frac{c_{\beta}}{4} \frac{1}{n^{3}} L_{2}^{\mathrm{opt}}(\bar{k})=-c_{\beta} \frac{1}{n^{3}}$ & $R_{2}^{\mathrm{opt}}(\bar{k})=\frac{2 c_{\delta}}{\gamma} \frac{1}{n}$ \\
\hline
\end{tabular}




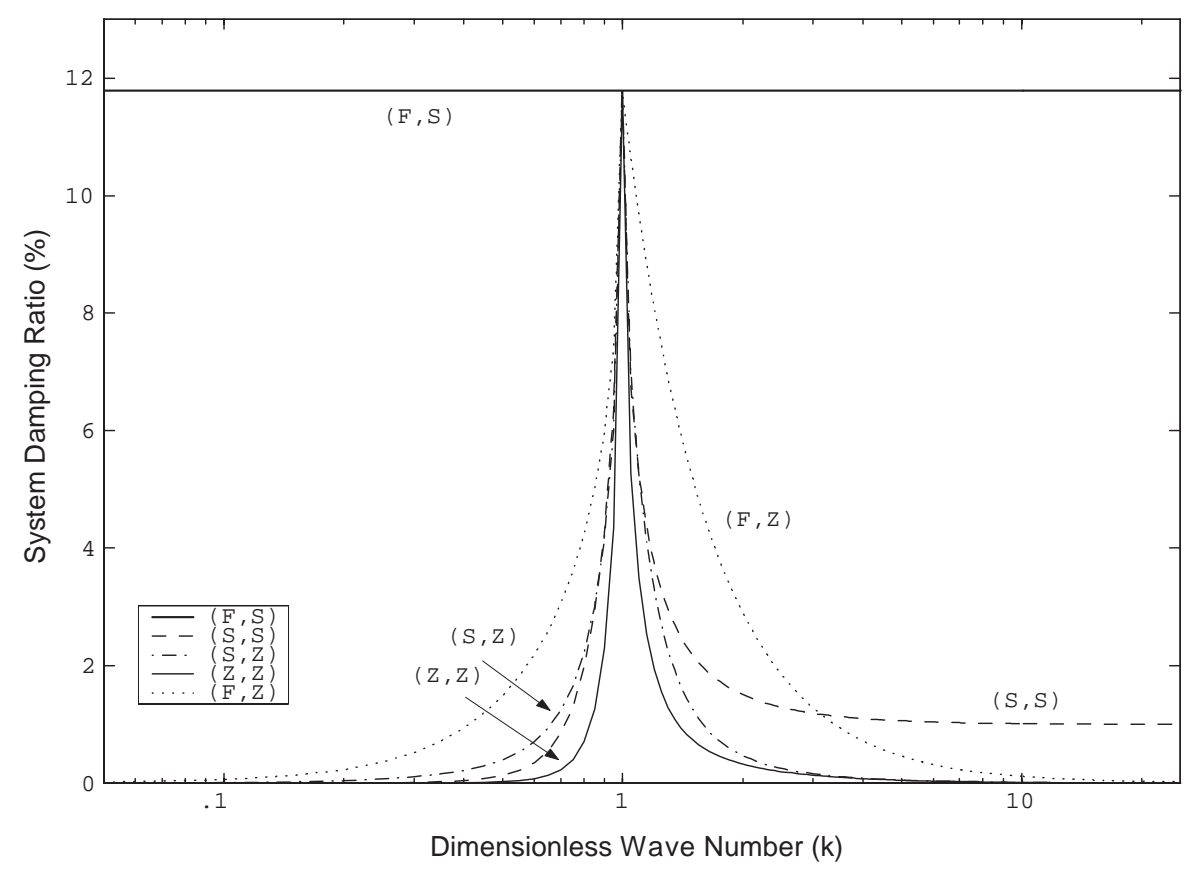

Fig. 7. System damping ratio $\zeta$ for the distributed vibration absorbers optimized for a dimensionless wave number $\bar{k}=1$.

The (F,S) network satisfies both conditions, therefore we expect that such a system can be optimized for all the wave numbers at the same time (this important result is confirmed by the plots in Figs. 6 and 7).

(3) Dependence on number of modules: The optimal inductance in each module is proportional to $n$ in the zeroth-order network, proportional to $1 / n$ in the second-order networks, and proportional to $1 / n^{3}$ in the fourth-order networks. Hence, their optimal values can be significantly decreased in second- and fourth-order networks by increasing the number of modules per unit of length. This fact can be relevant because the construction of high-value inductors implies non-trivial technological problems. Moreover, in the network with a secondorder dissipation, the optimal value of the parallel-connected resistances $R_{2}$ decreases for increasing $n$. Also this effect is desirable because the unavoidable parasite resistances of the electric components imply a technological upper bound on the value of $R_{2}$.

\subsubsection{Performance of optimal systems}

Once a system is optimized for a single wave number $\bar{k}$, it is interesting to study the corresponding damping properties for $k$-waves which are characterized by wave numbers $k$ different from $\bar{k}$.

Let us denote with $\beta_{i}^{\text {opt }}(\bar{k}), \delta_{j}^{\text {opt }}(\bar{k})$ the optimal damping and tuning parameters related to a generic $i$ th order electric network with a $j$ th order dissipation (refer to Eqs. (2a)-(2e)). As it is evident from Eq. (52) the corresponding optimal damping ratio is independent of the electric network. In order to point out the different damping performances shown by different distributed 
vibration absorbers, it is useful to define a function, named the characteristic decay time function, as follows:

$$
T_{\mathrm{opt}}(k, \bar{k}):=\frac{1}{P I\left(k, \beta_{i}^{\mathrm{opt}}(\bar{k}), \delta_{j}^{\mathrm{opt}}(\bar{k})\right)} .
$$

This furnishes the value of the characteristic (non-dimensional) decay time for a wave number $k$ in a structure, the electric circuitry of which is optimized for the wave number $\bar{k}$.

The optimal decay time function obtained for $\bar{k}=k$, i.e. the characteristic decay time obtained by optimizing the system for the current wave number $k$, can be evaluated explicitly from relationships (51):

$$
T_{\mathrm{opt}}(k, k)=\frac{2}{\gamma k^{2}} \text {. }
$$

Expression (54) depends only on the considered wave number and coupling coefficient and, as one could expect, the longer the wave number, the smaller the decay time. Hence, the same optimal decay time function may be attained regardless of the circuit topology. On the contrary, we anticipate that, when $k \neq \bar{k}$, the performance of the different PEM systems depends significantly on the chosen circuit topology (see e.g. the plots in the following Figs. 5-7).

Since the optimal decay time function depends on $k$ as governed by Eq. (54), it is convenient to define the following relative decay time function:

$$
\tilde{T}_{\text {opt }}(k, \bar{k}):=\frac{T_{\text {opt }}(k, \bar{k})}{T_{\text {opt }}(k, k)}=\frac{\gamma}{2} k^{2} T_{\text {opt }}(k, \bar{k})
$$

It can be interesting also to analyse the behaviour of the system damping ratio $\zeta(k, \bar{k})$ as a function of $k$ in a system optimized for the wave number $\bar{k}$. Note that this function is meaningful only when eigenvalues with non-vanishing imaginary part are present.

\subsection{Design of PEM beams}

In the present subsection, by considering a numerical example, the optimal electric parameters are found for a generic wave number $\bar{k}$ and the damping performance of the five network configurations are analysed and compared. Since we are studying the system described by the nondimensional differential equations (28), it is always possible to consider $\bar{k}=1$ (if $\bar{k}_{d}$ is the dimensional value of the wave number $\bar{k}$, it suffices to fix the scaling length $X_{0}=1 / \bar{k}_{d}$ ).

Let us consider a rectangular cross-section aluminium beam on which piezoelectric sheets made by the piezoelectric material PSI-5H-S4-ENH are bonded. In Table 5, the cross-section and material properties of the beam and of the piezoelectric sheets are reported. The longitudinal covering factor $\vartheta=0.9$ is fixed.

The homogenized material properties, the dimensionless coupling coefficient $\gamma$ and the constants $\left(c_{\beta}, c_{\delta}\right)$ can be evaluated from definitions (23), (24) and (25). Their numerical values are reported in Table 6. The corresponding dimensionless coupling coefficient is $\gamma=0.237$. The associated optimal damping ratio is $\zeta=11.78 \%$ (see Eq. (52)) and it is the same for the five vibration absorbers. 
Table 5

Aluminium beam and piezoelectric sheet cross-section dimensions and constitutive parameters

\begin{tabular}{lll}
\hline & Beam & PZT \\
\hline Width $\left(w_{b}, w_{p}\right)$ & $40 \times 10^{-3} \mathrm{~m}$ & $36.2 \times 10^{-3} \mathrm{~m}$ \\
Thickness $\left(h_{b}, h_{p}\right)$ & $4.0 \times 10^{-3} \mathrm{~m}$ & $0.267 \times 10^{-3} \mathrm{~m}$ \\
Young modulus $\left(Y_{b}, Y_{p}^{E}\right)$ & $70 \times 10^{9} \mathrm{~N} / \mathrm{m}^{2}$ & $62 \times 10^{9} \mathrm{~N} / \mathrm{m}^{2}$ \\
Mass density $\left(\rho_{b}, \rho_{p}\right)$ & $2700 \mathrm{~kg} / \mathrm{m}^{3}$ & $7800 \mathrm{~kg} / \mathrm{m}^{3}$ \\
Dielectric constant $\left(e_{33}^{T}\right)$ & - & $3800 \times \epsilon_{0}=3.36 \times 10^{-8} \mathrm{~F} / \mathrm{m}$ \\
Charge constant $\left(d_{31}\right)$ & - & $-320 \times 10^{-12} \mathrm{~m} / \mathrm{V}$ \\
\hline
\end{tabular}

Table 6

Homogenized constitutive parameters

\begin{tabular}{llllll}
\hline $\bar{g}_{m m}$ & $\bar{g}_{e e}$ & $\bar{g}_{m e}$ & $\bar{\sigma}$ & $c_{\beta}$ & $c_{\delta}$ \\
\hline $19.8 \mathrm{~N} / \mathrm{m}^{2}$ & $6.83 \times 10^{-6} \mathrm{~F} / \mathrm{m}$ & $2.76 \times 10^{-3} \mathrm{~N} / \mathrm{m}^{2} / \mathrm{V}$ & $0.568 \mathrm{~kg} / \mathrm{m}^{3}$ & $4.19 \times 10^{3} X_{0}^{3} \mathrm{H}$ & $24.8 \times 10^{3} X_{0} \Omega$ \\
\hline
\end{tabular}

Once the numerical value of $\gamma$ is known, it is possible to evaluate the decay time functions $T_{\text {opt }}(k, 1)$ and $\tilde{T}_{\text {opt }}(k, 1)$ by means of Eqs. (53) and (55). The corresponding plots for the five network configurations are reported in Figs. 5 and 6. In Fig. 7 we show also the dependence on $k$ of the system damping ratio defined by Eq. (46).

Figs. 6 and 7 show some important results. The system with (F, S)-network attains the optimal performance for all wave numbers: this means that

$$
\tilde{T}_{\text {opt }}(k, 1):=\frac{T_{\text {opt }}(k, \bar{k})}{T_{\text {opt }}(k, k)}=1
$$

for each $k$. This result agrees with those presented in Table 4, which shows that the optimal resistances and inductances are independent of $k$ for the $(\mathrm{F}, \mathrm{S})$-network. Furthermore, $(\mathrm{S}, \mathrm{S})$ network can appreciably damp free oscillations with $k>1$, although the corresponding damping ratio is much lower than the optimal one which is reached only for $k=\bar{k}=1$. Also this behaviour is in accordance with the relations for the optimal resistances and inductances in Table 4. Indeed, in $(\mathrm{S}, \mathrm{S})$-network the dissipation is always optimal, while the electromechanical resonant energy exchange is effective only for a narrowband of wave numbers.

It is important to recall that the proposed dynamic analysis is based on the homogenized beam model of the modular systems. As a consequence the presented results are meaningful only for wave numbers which are sufficiently small. Roughly speaking, the dimensionless wave number $k$ must be smaller than (i) the wave number $k_{w}=2 \pi /\left(w_{b} / X_{0}\right)$ corresponding to a wavelength equal to the beam width (we assume $h_{b}<w_{b}$ ), (ii) the wave number $k_{l}=\pi /\left(l_{b} / X_{0}\right)=n \pi$ corresponding to a wavelength equal to twice the longitudinal dimension of a single module. The first limit is an intrinsic drawback of the assumption of a one-dimensional beam model. The second restriction is related to the approximation implied by the homogenized model which is based on a second-order Taylor expansion of the state variables in each module. The latter limitation can also be regarded as a design relationship. Indeed, if one desires to realize a controller that is effective up to a 
dimensionless wave number $k_{\max }$, then a number of modules per characteristic length

$$
n>n_{\min }=\frac{k_{\max }}{\pi}
$$

must be used. The limitation above is a necessary condition only and it does not guarantee the effectiveness of the system for the wave number $k_{\max }$.

\subsection{Simply supported beam}

When a finite length beam is considered, the analysis of the time evolution of standing waves can be fruitfully applied for giving an analytical expression of the general solution to the corresponding boundary value problem. Hence, when the proper boundary conditions are introduced, it is possible to expand the solution of the system of partial differential equations (20) as a Fourier series of standing waves. For illustrative purposes, we analyse the particular case of a simply supported PEM beam, which allows a simple study of the electromechanical system. We denote with $l_{d}$ the beam length and with $l$ the corresponding non-dimensional expression. The simply supported PEM beam is modelled by the system of partial differential equations (20) subjected to the mechanical boundary conditions

$$
U(0, t)=U(l, t)=0, \quad U^{\prime \prime}(0, t)=U^{\prime \prime}(l, t)=0 .
$$

The choice of the corresponding electric boundary conditions is a design issue and should be finalized to the enhancement of the electromechanical coupling. The required boundary conditions are four for the fourth-order networks (F,Z) and (F,S), two for the second-order networks $(\mathrm{S}, \mathrm{Z})$ and $(\mathrm{S}, \mathrm{S})$ and zero for the zeroth-order network $(\mathrm{Z}, \mathrm{Z})$. In correspondence to the mechanical boundary conditions (58), we assume the electric boundary conditions

$$
\Psi(0, t)=\Psi(l, t)=0, \quad \Psi^{\prime \prime}(0, t)=\Psi^{\prime \prime}(l, t)=0
$$

for the fourth-order networks and

$$
\Psi(0, t)=\Psi(l, t)=0
$$

for the second-order networks (see also [9,7], respectively). The boundary modules which allow the realization of an approximate version of the boundary conditions (59) and(60) are reported in Fig. 8 .

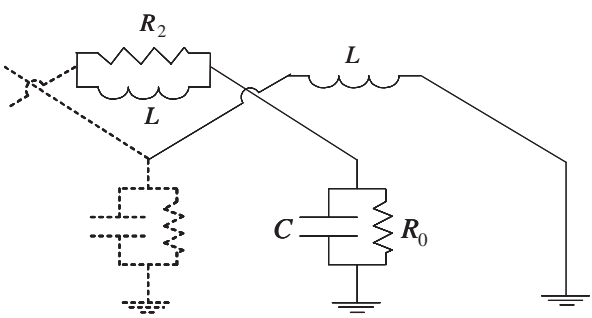

(a)

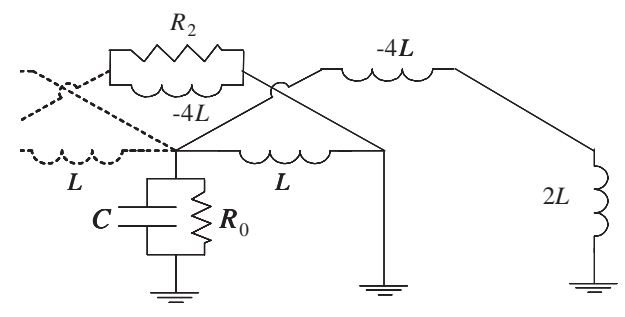

(b)

Fig. 8. Boundary module for (a) second-order and (b) fourth-order networks. 
The general solution of equation (20) with the simply supported boundary conditions can be formally expanded as follows:

$$
\mathbf{s}(X, t)=\sum_{i=1}^{\infty} \mathbf{s}_{i}(X, t)=\sum_{i=1}^{\infty} \mathrm{s}_{i}(t) \sin \left(i \pi \frac{X}{l}\right) .
$$

As it can be easily checked, each temporal evolution $\mathrm{s}_{i}(t)$ is independent and is governed by the system of two ordinary differential equations (28). Hence, the temporal evolution of $\mathbf{s}_{i}(X, t)$ is the same as that of a standing wave with a non-dimensional wave number $k=\alpha i$, with $\alpha \in \mathbb{R}$. In particular, if the scaling length $X_{0}=l_{d} / \pi$ is chosen, $\alpha=1$.

Each $s_{i}(t)$ evolves in time like a pair of electromechanical oscillators. As previously described, the parameters of the electric networks can be optimized only for a single $i$. The optimal numerical values and the corresponding damping performance for the $i$ th pair can be directly obtained from the table, relations (3) and Eq. (53) by replacing $k$ with $i$. As a numerical example, we consider a simply supported PEM beam with the geometrical and material properties given in Table 5. We report in Table 7 the damping ratios which are obtained for the first six mechanical modes when the distributed vibration absorbers are optimized for the first one $(\bar{k}=i=1)$. These values can be also read directly from the plot in Fig. 7. As a reference let us recall that the typical damping ratio due to internal dissipation for an aluminium beam is $0.1 \%$. As expected, the distributed vibration absorber $(\mathrm{F}, \mathrm{S})$ provides a simultaneous optimal damping of all the vibration modes. A remarkable multi-modal damping can be obtained also with the (S,S)-network.

The proposed optimization is based on the analysis of the free evolution of the PEM beams. However, it is relevant also to investigate the behaviour when an external mechanical excitation is applied. To this end, we consider the frequency response function (FRF) between the beam axis vertical displacement $U\left(X_{u}, t\right)$ at point $X_{u}$ and a concentrated transverse force applied at point $X_{f}$. In Fig. 9, the FRFs obtained with the various vibration absorbers are compared with the FRF related to the $(\mathrm{F}, \mathrm{S})$-network for $X_{u}=0.3 l$ and $X_{u}=0.7 l$. The scaling time is chosen in order to have a non-dimensional angular frequency $\omega_{i}=i^{2}$. The comments of the previous subsections can be adapted to the case of forced vibrations. In particular, we explicitly note that:

(1) these results should be interpreted considering that the systems are optimized such to minimize the characteristic decay time of the first mode during free oscillations;

(2) the FRFs have the same behaviour around $\omega=1$ for all systems, in accordance with the results shown in Figs. 6-7; and

Table 7

Characteristic damping ratios for $i=1, \ldots, 6$

\begin{tabular}{llllll}
\hline Network & $(\mathrm{Z}, \mathrm{Z})(\%)$ & $(\mathrm{S}, \mathrm{Z})(\%)$ & $(\mathrm{S}, \mathrm{S})(\%)$ & $(\mathrm{F}, \mathrm{Z})(\%)$ & $(\mathrm{F}, \mathrm{S})(\%)$ \\
\hline 1 & 11.78 & 11.78 & 11.78 & 11.78 & 11.78 \\
2 & 0.33 & 0.47 & 1.51 & 2.91 & 11.78 \\
3 & 0.13 & 0.16 & 1.19 & 1.29 & 11.78 \\
4 & 0.074 & 0.08 & 1.10 & 0.73 & 11.78 \\
5 & 0.047 & 0.05 & 1.06 & 0.46 & 11.78 \\
6 & 0.033 & 0.03 & 1.04 & 0.32 & 11.78 \\
\hline
\end{tabular}



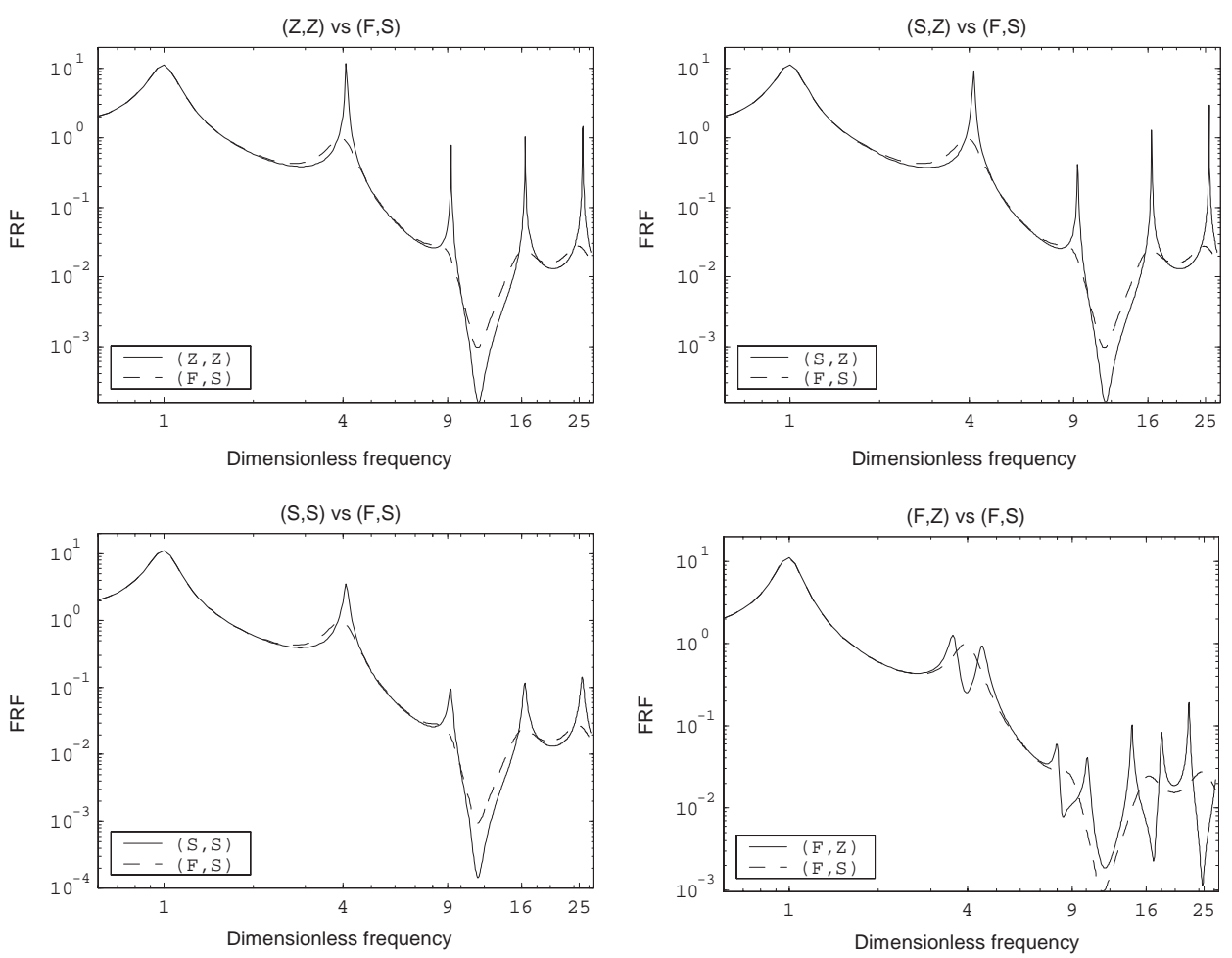

Fig. 9. Mechanical frequency response function for the vibration absorbers optimized to damp the free oscillations of the first mechanical mode. The response obtained by means of the fourth-order line (F, S) is compared with those related to the other networks. The plots correspond to the FRF between the transverse displacement at the axial point $X_{u}=0.3 l$ and a transverse force applied at the point $X_{f}=0.7 l$.

(3) the (F,S) network optimally reduces the FRF in correspondence to all the PEM beam resonances; also the (F, Z) network accomplishes a multi-modal tuning between the mechanical beam and the electric network, but the corresponding electric dissipation is optimal only for $\omega \simeq 1$; the $(\mathrm{S}, \mathrm{S})$-network introduces an appreciable damping at all natural frequencies, although it is optimally tuned only to the first mechanical mode.

In order to control the oscillations up to a given wave number, the PEM beam must be formed by a minimum number of modules per unit length, as ruled by relation (57). As a consequence, the numerical results presented above about the damping achieved for the first six terms of expansion (61) are not meaningful for less than six modules. In practice, since for acceptable results the lumped networks must emulate with a sufficient approximation the behaviour of a continuous system for the first six modes, it is appropriate to design a PEM beam with at least 12 modules. By assuming $n=12$, the optimal inductors and resistors for a beam of length $l_{d}=0.5 \mathrm{~m}$ are reported in Table 8. 
Table 8

Optimal inductors and resistors in each module for the simply supported PEM beam

\begin{tabular}{lll}
\hline Network & Optimal inductors & Optimal resistors $(\mathrm{k} \Omega)$ \\
\hline$(\mathrm{Z}, \mathrm{Z})$ & $L_{0}^{\mathrm{opt}}(\bar{k})=66.5 \mathrm{H}$ & $R_{0}^{\mathrm{opt}}(\bar{k})=32.1$ \\
$(\mathrm{~S}, \mathrm{Z})$ & $L_{2}^{\text {opt }}(\bar{k})=18.2 \mathrm{H}$ & $R_{2}^{\text {opt }}(\bar{k})=32.1$ \\
$(\mathrm{~S}, \mathrm{~S})$ & $L_{2}^{\text {opt }}(\bar{k})=18.2 \mathrm{H}$ & $R_{0}^{\text {opt }}(\bar{k})=32.1$ \\
$(\mathrm{~F}, \mathrm{Z})$ & $L_{1}^{\text {opt }}(\bar{k})=781 \mathrm{mH}, L_{2}(\bar{k})=-312 \mathrm{mH}$ & $R_{2}^{\text {opt }}(\bar{k})=8.80$ \\
$(\mathrm{~F}, \mathrm{~S})$ & $L_{1}^{\text {opt }}(\bar{k})=781 \mathrm{mH}, L_{2}(\bar{k})=-312 \mathrm{mH}$ & \\
\hline
\end{tabular}

\section{Conclusions}

In the present paper we proved that the concept of distributed piezoelectric vibration control is of interest in technological applications. We considered several electromechanical systems made of distributed piezoelectric transducers bonded on a beam and interconnected by properly designed electric networks. These systems were regarded as waveguides supporting electromechanical oscillations. The electric network interconnecting piezoelectric transducers were designed in order to enhance, by taking advantage of internal resonance effects, the transformation of mechanical energy into the electric form. In these networks, the mechanical energy is dissipated in dedicated resistors, which were chosen in order to obtain optimal vibration decay times. The performance of different circuit topologies for the interconnection of the piezoelectric transducers were compared and we showed that a passive controller able to simultaneously optimally damp all the mechanical vibration modes can be obtained.

The main results obtained for the control of the transverse vibrations of a beam are summarized in the following list.

(i) Every considered topology may be optimized to achieve the optimal damping performance for a single wave number. This optimal decay time is the same for all the circuit topologies and depends only on the electromechanical coupling coefficient.

(ii) The performance of the compared circuits optimized for a single wave number varies significantly when standing waves of different wave numbers are considered.

(iii) If a distributed vibration absorber with proper dynamic properties is considered, its optimal electric parameters are independent of the wave number. To this purpose, the dissipation term associated to the resistors must appear in the homogeneous equations with a second-order spatial derivative, the "electric stiffness" term due to the inductors, with a fourth-order spatial derivative. Hence, the fourth-order transmission line with second-order dissipation $(F, S)$ supplies the optimal decay time for all the wave numbers.

(iv) The nominal value of the inductances in each module depends on the number $n$ of modules (i.e. transducers) per unit length in different ways and this dependence is affected by the chosen interconnecting circuit topology. The most convenient circuit is again the fourth-order transmission line: in this case the inductances in each module are proportional to $1 / n^{3}$. This is relevant because the lower the inductances are, the easier is their technological construction with electr(on)ic components. 
(v) The piezoelectric controller obtained with the $(F, S)$ network is robust under forcing frequency shifts, accomplishing a broadband optimal damping. This point was illustrated by means of a numerical example of a simply supported PEM beam, which may be useful to the design of a demonstration prototype.

The proposed damping devices can be actually constructed. Although technological aspects have not been detailed in this paper, it should be noted that the required high or negative inductances are feasible only using electronic active filters. The technological and theoretical problems to be solved in this context are related to the synthesis of equivalent electric networks which are completely passive.

\section{Acknowledgements}

The present research has been partially supported by MIUR ("Ministero per l'Innovazione, l'Università e la Ricerca Scientifica"-Fondi Ricerca PRIN 2001097882_003 "Sintesi di circuiti piezoelettrici e tecniche di disaccoppiamento per il controllo di vibrazioni meccaniche") and by the University of Rome "La Sapienza"-Fondi Ateneo 1999/2000. The partial support of the Engineering Science and Mechanics Department of Virginia Polytechnic Institute and State University is gratefully acknowledged by the authors.

\section{References}

[1] J.P. Den Hartog, Mechanical Vibrations, Dover Publications, New York, 1953.

[2] N.W. Hagood, A. von Flotow, Damping of structural vibrations with piezoelectric materials and passive electric networks, Journal of Sound and Vibrations 146 (2) (1991) 243-268.

[3] V. Steffen Jr., D.J. Inman, Optimal design of a piezoelectric material for vibration damping in mechanical systems, Journal of Intelligent Material Systems and Structures 10 (1999) 945-955.

[4] P. Bisegna, G. Caruso, D. Del Vescovo, S. Landini, Simulation of vibrations passive damping of a piezoactuated cantilever plate, Annual Conference of Italian Society for Computer Simulations-ISCS, Rome, 1999.

[5] J.J. Hollkamp, Multimodal passive vibration suppression with piezoelectric materials and resonant shunts, Journal of Intelligent Material Systems and Structures 5 (1994) 49-57.

[6] F. dell'Isola, S. Vidoli, Continuum Modeling of piezoelectro mechanical truss beams: an application to vibration damping, Archive Applied Mechanics 68 (1998) 1-19.

[7] S. Vidoli, F. dell'Isola, Modal coupling in one-dimensional electromechanical structured continua, Acta Mechanica 141 (2000) 1-2.

[8] S. Vidoli, F. dell'Isola, Vibration control in plates by uniformly distributed PZT actuators interconnected via electric networks, European Journal of Mechanics/A Solids 20 (2001) 435-456.

[9] S. Alessandroni, F. dell'Isola, M. Porfiri, A revival of electric analogs for vibrating mechanical systems aimed to their efficient control by PZT actuators, International Journal of Solids and Structures 39 (20) (2002) 5295-5324.

[10] O. Thorp, M. Ruzzene, A. Baz, Attenuation and localization of wave propagation in rods with periodic shunted piezoelectric patches, Journal of Smart Materials and Structures 10 (2001) 979-989.

[11] S. Yarlagadda, G.A. Lesieutre, S. Yoshikawa, J. Witham, Resistively shunted piezocomposites for passive vibration damping, Proceedings of the AIAA/ASME/AHS Adaptive Structures Forum, Salt Lake City, UT, 1996, pp. 217-227.

[12] E. Canon, M. Lenczner, Modelling of thin elastic plates with small piezoelectric inclusions and distributed electronic circuits. Models for inclusions that are small with respect to the thickness of the plate, Journal of Elasticity 55 (1999) 111-141. 
[13] Heun-Ho Rew, In Lee, Adaptive shunting for vibration control of frequency-varying structures, Journal of Guidance, Control and Dynamics 24 (6) (2001).

[14] J.J. Hollkamp, T.F. Starchville, A self-tuning piezoelectric vibration absorber, Journal of Intelligent Material Systems and Structures 5 (4) (1994) 559-566.

[15] W.J. Karplus, W.W. Soroka, Analog Methods. Computation and Simulation, McGraw-Hill, New York, 1959.

[16] L. Brillouin, Wave Propagation in Periodic Structures. Electric Filters and Crystal Lattices, McGraw-Hill, New York, 1946.

[17] U. Andreaus, F. dell'Isola, M. Porfiri, Piezoelectric passive distributed controllers for beam flexural vibrations, to appear.

[18] C. Woźniac, Refined macro-dynamics of periodic structures, Archive of Mechanics 45 (1993) 295-304.

[19] P. Germain, The method of virtual power in continuum mechanics. Part 2: microstructure, SIAM Journal of Applied Mathematics 25 (3) (1973) 556-575.

[20] G.A. Maugin, The method of virtual power in continuum mechanics: application to coupled field, Acta Mechanica 3 (1980) $1-70$.

[21] F. dell'Isola, L. Rosa, C. Woźniac, A micro-structured continuum modelling of compacting fluid-saturated grounds: the effects of pore-size scale parameter, Acta Mechanica 127 (1998) 165-182.

[22] C. Maurini, Electromechanical coupling of distributed piezoelectric transducers for passive damping of structural vibrations: comparison of network configurations, 2002, Master Thesis, Virginia Polytechnic Institute and State University, 2002.

[23] IEEE Standards on piezoelectricity, ANSI/IEEE Std 176-1987.

[24] A.C. Eringen, G.A. Maugin, Electrodynamics of Continua, Vols. I-II. Springer-Verlag, New York, 1990.

[25] T. Ikeda, Fundamentals of Piezoelectricity, Oxford University Press, Oxford, 1990.

[26] D.A. Saravanos, P.R. Heyliger, Mechanics and computational models for laminated piezoelectric beams, plates and shells, Applied Mechanical Review 52 (10) (1999) 305-320.

[27] L. Perko, Differential Equations and Dynamical Systems, Texts in Applied Mathematics, Springer-Verlag, New York, 1996.

[28] R. Courant, D. Hilbert, Methods of Mathematical Physics, Vol. 2, Interscience Publishers, Inc., New York, 1953. 